\title{
Convergence of the Viscosity Method for Isentropic Gas Dynamics
}

\author{
Ronald J. DiPerna \\ Department of Mathematics, Duke University, Durham, NC 27706, USA
}

\begin{abstract}
A convergence theorem for the method of artificial viscosity applied to the isentropic equations of gas dynamics is established. Convergence of a subsequence in the strong topology is proved without uniform estimates on the derivatives using the theory of compensated compactness and an analysis of progressing entropy waves.
\end{abstract}

\section{Introduction}

We are concerned with the zero diffusion limit for hyperbolic systems of conservation laws. The general setting is provided by a system of $n$ equations in one space dimension,

$$
u_{t}+f(u)_{x}=0 \text {, }
$$

where $u=u(x, t) \in R^{n}$ and $f$ is a smooth nonlinear map defined on a region $\Omega$ of $R^{n}$. The zero diffusion limit is concerned with the convergence of approximate solutions to (1.1) generated by parabolic regularization. In this paper we shall deal with the Cauchy problem for diffusion processes of the classical form

$$
u_{t}+f(u)_{x}=\varepsilon D(u)_{x x},
$$

and we shall establish, in particular, a convergence theorem for the method of artificial viscosity applied to the isentropic equations of gas dynamics with a polytropic equation of state

$$
\begin{aligned}
\varrho_{t}+(\varrho u)_{x} & =0, \\
(\varrho u)_{t}+\left(\varrho u^{2}+p\right)_{x} & =0, \quad p=\text { const } \varrho^{\gamma} .
\end{aligned}
$$

The conservation laws of mass and momentum (1.3) may, of course, be formulated in terms of the primitive densities $\varrho$ and $m=\varrho u$ to yield the form (1.1):

$$
\begin{gathered}
\varrho_{t}+m_{x}=0, \\
m_{t}+\left(m^{2} / \varrho+p\right)_{x}=0 .
\end{gathered}
$$


We shall consider the Cauchy problem with smooth data in $L^{\infty}(R)$ that approaches a constant state $(\bar{\varrho}, \bar{u})$ at infinity and satisfies

$$
\varrho_{0}(X) \geqq \delta>0,
$$

and prove that the solutions $\left(\varrho^{\varepsilon}, u^{\varepsilon}\right)$ of the process

$$
\begin{aligned}
\varrho_{t}+(\varrho u)_{x} & =\varepsilon \varrho_{x x}, \\
(\varrho u)_{t}+\left(\varrho u^{2}+p\right)_{x} & =\varepsilon(\varrho u)_{x x},
\end{aligned}
$$

based on equal diffusion rates for mass and momentum, converge to a globally defined distributional solution $(\varrho, u)$ of $(1.3)$ satisfying $0 \leqq \varrho \leqq$ const, $|u| \leqq$ const, where the constants depend only on the adiabatic exponent $\gamma$ and the $L^{\infty}$ norm of the initial data $\left(\varrho_{0}, u_{0}\right)$. After modification on a set of measure zero, the solution $(\varrho, u)$ is continuous in the spatial weak topology as a function of time: for every test function $\phi$, the average values $\int \phi(x) \varrho(x, t) d x, \int \phi(x) \varrho u(x, t) d x$ are continuous in $t$ and converge to the data $\int \phi(x) \varrho_{0}(x) d x, \int \phi(x) \varrho_{0} u_{0}(x) d x$ as $t$ approaches zero.

Several assumptions are adopted for technical convenience. First, we assume a uniform lower bound (1.4) on the initial density $\varrho_{0}$. In this situation it is particularly simple to construct globally defined smooth solutions to the system

$$
\begin{aligned}
\varrho_{t}+m_{x} & =\varepsilon \varrho_{x x}, \\
m_{t}+\left(m^{2} / \varrho+p\right) & =\varepsilon m_{x x},
\end{aligned}
$$

since (1.6) provides a standard uniformly parabolic representation of (1.5). It can be shown that cavities do not develop in finite time in a viscous gas, i.e.

$$
\varrho^{\varepsilon}(x, t) \geqq \delta^{\varepsilon}(t)>0
$$

for an appropriate function $\delta^{\varepsilon}$, cf. Sect. 4. In the presence of an a priori lower bound of the form (1.7), it is a straightforward process to continue a local solution of (1.6) in time; one need only appeal to the invariant quadrants in the plane of Riemann coordinates to establish an $L^{\infty}$ estimate independent of time (and of $\varepsilon$ ). Second, we assume that the initial data $\left(\varrho_{0}, u_{0}\right)$ lies in $C^{2}(R)$ and rapidly approaches a constant state $(\bar{\varrho}, \bar{u})$ at infinity in the sense that the difference $\left(\varrho_{0}-\bar{\varrho}, u_{0}-\bar{u}\right)$ lies in $H^{2}(R)$. In the presence of this type of regularity and decay, one may easily work on the line and avoid finite boundary terms which arise, for example, in the analysis of the entropy field. Third, we shall restrict attention, in the final stages of the argument, to the physically relevant sequence of adiabatic exponents, namely $\gamma=1+2 / n$, where $n$ denotes the number of degrees of freedom of the molecules. We recall that the integer $n$ is necessarily greater than or equal to three due to the presence of three translational degrees of freedom. In the special case where $n$ is an odd integer, the Riemann function for the compatibility equation linking generalized entropy with its flux reduces to a polynomial and the basic computations are simplified.

Before discussing the proof we shall remark on the relevant background. One natural strategy for proving convergence as the diffusion parameter $\varepsilon$ vanishes is to seek uniform estimates on the amplitude and derivatives of the approximate solutions $u^{\varepsilon}$ and then appeal to a compactness argument in order to extract a 
strongly convergent subsequence and pass to the limit in the nonlinear flux $f$. In the context of conservation laws, the spatial $L^{\infty}$ and total variation norms provide a natural pair of metrics to investigate stability (in the sense of uniform boundedness) of families of exact and approximate solutions. The $L^{\infty}$ norm provides an appropriate measure of the solution amplitude while the total variation norm provides an appropriate measure of the solution gradient. Uniform control on both metrics guarantees the existence of a subsequence converging pointwise a.e. The relevance of these norms for strictly hyperbolic systems (1.1.) is indicated by Glimm's fundamental theorem [11] which establishes $L^{\infty}$ and total variation stability for the random choice difference approximations in the case of small initial data. We remark that it remains an open problem to establish the corresponding stability estimates for either classical diffusion processes or finite difference schemes that are conservative in the sense of Lax and Wendroff [15], even in the setting of small data.

An alternative approach to the convergence problem, which is used here, is to established just $L^{\infty}$ stability and pass to the limit with the aid of the theory of compensated compactness $[17,18,22,23]$. Regarding previous work in this direction, we recall that Tartar [22] has established a new convergence theorem for the viscosity method applied to a scalar law in one space dimension using only the uniform $L^{\infty}$ bound afforded by the maximum principle. The analysis employs the weak topology and averaged quantities. One of the main tools is provided by the following result which express composite weak limits as expected values. Suppose $v_{k}: R^{m} \rightarrow R^{n}$ is an arbitrary sequence of functions uniformly bounded in $L^{\infty}$. One may extract a subsequence, still labelled $v_{k}$, which converges in the weakstar topology:

$$
\int_{\Omega} v(y) d y=\lim _{k \rightarrow \infty} \int_{\Omega} v_{k}(y) d y,
$$

for all measurable $\Omega$ in $R^{m}$. By passing to a further subsequence one may assert the existence of a family of probability measures over the target space $R^{n}$, index by points of the domain space $R^{m}, v_{y}=v_{y}(\lambda), \lambda \in R^{m}$ with the following property. For all continuous real-valued maps on $R^{n}$, the composite limit exists in the weak-star topology and coincides almost everywhere with the expected of value $g$ :

$$
\begin{gathered}
g_{\infty}=\lim _{k \rightarrow \infty} g\left(v_{k}\right) \text { weak*, } \\
g_{\infty}(y)=\int_{R^{m}} \mathrm{~g}(\lambda) \mathrm{d} v_{y}(\lambda) \text { a.e. in } y .
\end{gathered}
$$

It follows that the deviation between weak and strong convergence is estimated by the diameter of the support of the representing measure $v_{y}$; in particular the sequence converges strongly if and only if $v_{y}$ reduces to a point mass.

In the setting of a scalar equation Tartar has shown that the measure $v_{(x, t)}$ associated with a sequence of solutions $u^{\varepsilon}$ to the equation, $u_{t}+f(u)_{x}=\varepsilon u_{x x}$, is concentrated on an interval where $f$ is affine. In the case where $f$ is not affine on any interval, for example, in the genuinely nonlinear case $f^{\prime \prime} \neq 0$, the measure $v_{(x, t)}$ reduces to a point mass and the convergence becomes strong. In the setting of strictly hyperbolic systems of two equations it has been shown [10] that the 
measure $v_{(x, t)}$ associated with proper diffusion processes reduces to a point mass if the characteristic speeds are genuinely nonlinear in the sense of Lax [13]: if $u^{\varepsilon}$ is a sequence of uniformly bounded solutions to a $2 \times 2$ parabolic system of the form $u_{t}$ $+f(u)_{x}=\varepsilon D u_{x x}$, for which the diffusion matrix $D$ induces correct entropy production, then there exists a subsequence $u^{\varepsilon_{k}}$ that converges pointwise a.e. to a solution of the corresponding hyperbolic system. The general conjecture is that the measure associated with approximate solutions generated by a method which respects the entropy condition either reduces to a point mass or concentrates itself on a set whose geometry permits the continuity of $f$ with respect to weak limits. In addition to diffusion processes, this conjecture has been established for a class of first order accurate conservative finite difference schemes including the LaxFriedrichs scheme and Godunov's scheme, applied to strictly hyperbolic genuinely nonlinear systems of two equations [10].

We note that it remains an open problem to establish a uniform $L^{\infty}$ bound for diffusion methods and classical difference schemes. Experience with the exact solution of (1.1) leads one to expect that, at the very least, initial data with small oscillation generates a solution with uniformly small oscillation. This type of behavior has been verified for the random choice difference approximations in the setting of strictly hyperbolic genuinely nonlinear systems of two equations [12]. The only pointwise bounds currently available for diffusion methods and the difference scheme are those derived for $2 \times 2$ systems using invariant regions. They require equal diffusion rates; one observes that the invariant quadrants for the exact hyperbolic solution operator viewed in the plane of Riemann coordinates are preserved by precisely those approximation methods which are based on equal rates of diffusion. This fact motivates our use of the method of artificial viscosity (1.6).

In this paper we are motivated partly by the problem of establishing existence of solutions to systems of conservation laws. The first large data existence theorem was obtained by Nishida [19] for the isothermal equations of gas dynamics, $p=$ const $\varrho$, using the random choice method. Large data theorems have also been obtained for the isentropic and non-isentropic equations of gas dynamics with a polytropic equation of state in the case where the initial data is restricted to prevent the development of cavities. We refer the reader to $[9,18-20]$ in connection with gas dynamics and to $[2,8]$ for special systems. The relevant analysis in the aforementioned papers involves estimates on local wave interactions, specifically estimates relating incoming and outgoing wave magnitudes in a binary interaction. The difficulty in bounding the total variation norm at low densities arises from the fact that the coupling between characteristic fields increases as $\varrho$ decreases. This increased coupling is a reflection of the fact that both strict hyperbolicity and hyperbolicity are lost at the vacuum, i.e. the eigenvalues and eigenvectors coalesce on the boundary of the state space, namely, the line $\varrho=0$. For comparison we note that a large data theorem has been obtained using compensated compactness for the equations of elasticity in the setting of a hard spring [10]. In that case one deals with distinct eigenvalues with a linear degeneracy in the interior of the state space. The large data existence problem arises in a variety of contexts. Concerning basic work on problems with degenerate eigenvalues we refer the reader to [24-26]. 
We shall conclude with a discussion of the convergence proof for system (1.6). Our analysis involves a study of entropy waves in the state space $R^{2}$. We recall that in the setting of a strictly hyperbolic system the representing measure $v_{(x, t)}$ is restricted by a commutativity relation that is derived from an analysis of the entropy fields and a basic continuity theorem of Tartar [20] and Murat [14] for bilinear maps in the weak topology. Specifically, $v$ commutes with an antisymmetric form acting on entropy pairs $(\eta, q)$, i.e. solutions of the linear hyperbolic system which links generalized entropy $\eta$ to its flux $q$ :

$$
\left\langle v, \eta_{1} q_{2}-\eta_{2} q_{1}\right\rangle=\left\langle v, \eta_{1}\right\rangle\left\langle v, q_{2}\right\rangle-\left\langle v, \eta_{2}\right\rangle\left\langle v, q_{1}\right\rangle
$$

for all solutions $\left(\eta_{j}, q_{j}\right)$ to

$$
\nabla \eta \nabla f=\nabla q .
$$

Tartar conjectured that any probability measure $v$ that satisfies (1.8) for all entropy pairs must reduce to a point mass if the eigenvalues are not degenerate on a large set. This conjecture has been verified for scalar equations [20], for a broad class of genuinely nonlinear $2 \times 2$ systems and for the special $2 \times 2$ system in elasticity which admits a single curve of degeneracy in the state space [10]. In order to establish (1.8) it is virtually necessary to prove that the divergence of the approximate entropy fields $\left(\eta_{j}\left(u^{\varepsilon}\right), q_{j}\left(u^{\varepsilon}\right)\right)$ lies in a compact subset of the negative Sobolev space $H^{-1}$. In the setting of the viscosity method applied to a scalar law, Tartar [20] verified the $H^{-1}$ condition for all entropy pairs using Murat's lemma [18] on the positive cone of $\mathrm{H}^{-1}$. For the viscosity method and first order difference schemes applied to $2 \times 2$ systems, the $H^{-1}$ condition has also been established for all entropy pairs with the aid of Murat's lemma [10]. In this paper we shall establish the $H^{-1}$ condition and therefore (1.8) for a restricted class of entropy pairs and show it forces the reduction of $v$ to a point mass.

Section 2 contains an analysis of the entropy waves for the equations of isentropic gas dynamics. In the case of a polytropic equation of state we show that the governing equation (1.9) for the entropy pair $(\eta, q)$ reduces to the classical EPD equation, Euler-Poisson-Darboux. We recall that in [10] the reduction property for the measure associated with nondegenerate $2 \times 2$ systems was established using the progressing entropy waves introduced by Lax [14]. Unfortunately, the coefficients of the formal asymptotic series for these progressing waves diverge as the eigenvalues coalesce at the vacuum, and one is required to entertain a different construction procedure. In Sect. 2 we construct progressing EPD waves by two different methods. We show that the EPD equation is of Fuchsian type and admits two distinct classes of waves (weak and strong) characterized by the nature of their algebraic singularity along the singular line $\varrho=0$ at the boundary of the state space. We prove that the weak entropy fields lie in a compact subset of $H^{-1}$ by showing that the mechanical energy is a convex weak wave whose Hessian dominates the Hessian of all weak waves at low density. On the other hand, as the density $\varrho$ vanishes, the strong waves evolve into saddle surfaces whose Hessian diverges at a rate which is an order of magnitude faster than the mechanical energy. We conjecture that the strong entropy fields do not lie in a compact subset of $H^{-1}$ as the viscosity parameter $\varepsilon$ vanishes. Using only the weak entropy pairs we show that $v$ reduces to a point mass. It is feasible that one could establish the 
commutativity relation (1.8) for all entropy pairs, weak and strong, using a truncation or smoothing procedure near the vacuum $\varrho=0$. Establishing (1.8) for all pairs would allow one to combine the analysis of [10] and Sect. 2 in a straightforward way to prove the reduction property for $v$. We remark that the long range goal of our program is to prove that $v$ reduces to a point mass for systems of conservation laws which switch from hyperbolic to elliptic type across a (parabolic) line. In the setting of mixed equations the first step of our approach is to establish the reduction property using only (1.8) acting on pairs which vanish on the transition line; the analysis developed in Sect. 3 has a dual purpose. In this paper we shall establish the reduction property for a polytropic gas in the case where $\gamma=1+2 / n, n$ and odd integer $>3$. The refinements needed to treat the general case $1<\gamma \leqq 5 / 3$ will be presented in a forthcoming paper on systems of mixed type.

In Sect. 3 the reduction property is established by studying the regularity of the measure $v$. The basic idea is to analyze the subspace of waves on which the antisymmetric form

$$
B=B\left(\eta_{1}, q_{1} ; \eta_{2}, q_{2}\right)=\eta_{1} q_{2}-\eta_{2} q_{1},
$$

is definite. Our study of elliptic systems in two independent variables has shown that $B$ is definite on a broad class of pairs $(\eta, q)$. Indeed, it can be shown that the coercivity of $B$ is equivalent to the ellipticity of a system of $n$ conservation laws in two independent variables. Although $B$ is indefinite for a hyperbolic system, it admits a small coercive subspace defined by the Goursat operator. In Sect. 3 we show that the coercive structure of $B$ on special Goursat waves allows one to differentiate $v$ in the Lebesgue sense and show that the derivative vanishes except at one point. Technically the differentiation process is carried out using pairs of progressing waves whose leading term is coercive with respect to $B$. This analysis bears out the following principle: for a general probability measure, the commutativity relation (1.8) represents an imbalance of regularity; the operator on the left is more regular than the one on the right due to cancellation, a fact which can only be resolved if $v$ reduces to a point mass. We shall expand on this remark in Sect. 3. In the following sections we shall assume that the reader is familiar with the arguments of [10].

We shall conclude with a few remarks on recent progress and open problems dealing with the representing measure $v$. Tartar [23] has recently established a definitive local reduction theorem for the measure $v$ associated with a general elliptic system in several dimensions : if the support of $v$ is sufficiently small, then $v$ is a point mass. The proof is based on an analysis of bilinear forms which are lower semicontinuous in the weak topology. In this connection we recall that the commutativity relation (1.8) expresses the fact that the antisymmetric inner product of two entropy pairs is continuous in the weak topology. The relation (1.8) is derived from the basic div-curl continuity lemma of Tartar and Murat. We have shown that the weak continuity expressed by (1.8) is sufficient to reduce $v$ globally for non-degenerate $2 \times 2$ hyperbolic systems and for the degenerate $2 \times 2$ system of gas dynamics, and locally for general $n \times n$ elliptic systems in two variables. Tartar has shown that the corresponding inequalities for lower semicontinuous forms are sufficient to reduce $v$ locally for general $n \times n$ elliptic systems in several variables. 
In the setting of two variables the available class of lower semicontinuous bilinear forms and continuous bilinear forms coincide.

Open problems remain for degenerate $2 \times 2$ hyperbolic systems and for nondegenerate $n \times n$ hyperbolic systems with $n>2$. In the latter case there typically exists at most one nonlinear entropy field and a new strategy may be needed.

Regarding work on the weak topology in the elliptic setting of mechanics we refer the reader to Ball [1-3] and Dacorogna [5-7].

\section{Entropy Waves}

This section is concerned with entropy waves for isentropic gas dynamics. We shall begin with some general remarks concerning systems of $n$ equations,

$$
u_{t}+f(u)_{x}=0 .
$$

We recall that a pair $(\eta, q)$ of real-valued maps on the state space $R^{n}$ is an entropy pair for (2.1) if all smooth solutions satisfy an additional conservation law, $\eta(u)_{t}+q(u)_{x}=0$. A necessary and sufficient condition for the existence of a pair $(\eta, q)$ is given by the compatibility condition

$$
\nabla \eta \nabla f=\nabla q,
$$

on $R^{n}$. If $f$ is hyperbolic, (2.2) constitutes a linear hyperbolic system of $n$ equations in two unknowns whose characteristic form

$$
r_{j} \cdot\left(\lambda_{j} \nabla \eta-\nabla q\right)=0, \quad j=1,2,
$$

is derived by dotting with the right eigenvectors of the Jacobian of $f: \nabla f r_{j}=\lambda_{j} r_{j}$. The characteristic curves of (2.3) are the integral curves of the vector fields $r_{j}$. In the special case $n=2$, it is convenient to introduce a coordinate system of Riemann invariants $(w, z)$,

$$
\begin{array}{ll}
r_{1} \circ \nabla w=0, & \partial / \partial w=r_{2} \cdot \nabla, \\
r_{2} \cdot \nabla z=0, & \partial / \partial z=r_{1} \cdot \nabla,
\end{array}
$$

which transforms (2.3) into

$$
\lambda_{1} \eta_{z}=q_{z}, \quad \lambda_{2} \eta_{w}=q_{2} .
$$

One may work equally well with a second order equation for $\eta$ by taking the curl of $(2.3)$ :

$$
\operatorname{curl}(\nabla \eta \nabla f)=0 \text {. }
$$

The hyperbolicity of (2.5) is easily recognized in the characteristic variables after cross differentiation:

$$
\begin{aligned}
\left(\lambda_{1} \eta_{z}\right)_{2} & =\left(\lambda_{2} \eta_{w}\right)_{z}, \\
\left(\lambda_{1}-\lambda_{2}\right) \eta_{z w}+\lambda_{1 w} \eta_{z}-\lambda_{2 w} \eta_{w} & =0 .
\end{aligned}
$$


In the setting of isentropic gas dynamics, the Riemann invariants and eigenvalues may be expressed explicitly in terms of the sound speed $c=\sqrt{p^{\prime}}$ :

$$
\begin{array}{ll}
w=u+\int c / \varrho d \varrho, & \lambda_{2}=u+c \\
z=u-\int c / \varrho d \varrho, & \lambda_{1}=u-c .
\end{array}
$$

For a polytropic gas $p=\varrho^{\gamma / \gamma}$, the eigenvalues are linear combinations of algebraic Riemann invariants:

$$
\begin{aligned}
& w=u+\varrho^{\theta} / \theta, \quad \lambda_{2}=\frac{(\gamma+1)}{4} w+\frac{(3-\gamma)}{4} z, \\
& z=u-\varrho^{\theta} / \theta, \quad \lambda_{1}=\frac{(\gamma+1)}{4} z+\frac{(3-\gamma)}{4} w,
\end{aligned}
$$

where $\theta=(\gamma-1) / 2$. We have normalized the pressure for notational convenience.

One easily verifies that the characteristic form of the entropy equation (2.6) becomes the Euler-Poisson-Darboux equation

$$
\eta_{z w}-\frac{\lambda}{(w-z)}\left(\eta_{w}-\eta_{z}\right)=0
$$

with index $\lambda=-\frac{1}{2}(3-\gamma) /(\gamma-1)$. The limiting behavior of EPD solutions at the vacuum $w=z$, depends upon the index $\lambda$. In the setting of a polytropic gas, $\lambda$ may be expressed in terms of the number of degrees of freedom of the molecules. We have $\gamma=1+2 / n,-\lambda=(n-1) / 2$, where $n$ is an integer $\geqq 3$.

Progressing waves for the EPD equation may be introduced in several ways. One may apply the separation of variables procedure in $(\varrho, u)$ coordinates to the equation,

$$
\eta_{\varrho \varrho}=d^{2} \eta_{u u}, \quad d=c / \varrho \text {. }
$$

We note that $\eta(w, z)$ solves EPD if and only if $\eta\left(u+\int c / \varrho, u-\int c / \varrho\right)$ solves (2.9). Specifically, if $k$ denotes a constant, the function $\eta=h(\varrho) e^{k u}$ solves (2.9) provided

$$
h^{\prime \prime}-k^{2} d h=0 \text {. }
$$

The Eq. (2.10) for density dependence may be transformed into a standard Fuchsian equation using the change of variables $a(\varrho)=\varrho^{(1-\theta) / 2}, r=k \varrho^{\theta} / \theta$. A simple calculation shows that $h=a(\varrho) \psi(r)$ solves $(2.10)$ if and only if

$$
\psi^{\prime \prime}=\left(1+\mu / r^{2}\right) \psi=0,
$$

when $\mu=\left(1-\theta^{2}\right) / 4 \theta^{2}$. Here we are working in the plane of Riemann invariants rotated by 45 degrees : $u$ and $\varrho^{\theta} / \theta$ are half the sum and difference of the invariants.

We note in passing that for a general equation of state $p=p(\varrho)$, the substitution $r=k(w-z)$ leads to a second order equation for $\psi$ with a variable coefficient. We expect that the program below can be carried out if $p$ acts like an algebraic function near $\varrho=0$.

In the case of a polytropic gas the appropriate ansatz for a progressing wave is provided by $\eta=h e^{k u}, q=u \eta+g e^{k u}$, where $h$ solves $(2.10)$ and $g(\varrho)$ is determined from $h$ by the formula $k g=\varrho h^{\prime}-h$. In the factored form,

$$
\eta=a \psi e^{k u}, \quad q=u \eta+a \tilde{g} e^{k u},
$$


the coefficient $\psi$ solves (2.11) while $\tilde{g}$ is determined from $\psi$ by the formula

$$
k \tilde{g}(r)=\theta r \psi^{\prime}-\frac{(1+\theta)}{2} \psi .
$$

Substituting (2.13) into (2.12) yields a formula for the flux in terms of the entropy, for each choice of $\psi$, namely

$$
q=\eta\left(u+c \psi^{\prime} / \psi-(1+\theta) / 2 k\right)
$$

In order to analyze the limiting behavior of the waves (2.12) at the vacuum, one may appeal to the method of Frobenius which, in this case, solves (2.11) with a series of the form

$$
\psi=\sum_{n \geqq j} \gamma_{n} r^{n} .
$$

The indicial equation has two distinct roots with corresponding independent solutions

$$
\begin{array}{ll}
j_{+}=(\theta+1) / 2 \theta, & j_{-}=(\theta-1) / 2 \theta, \\
\psi_{+}=r^{j+} \phi_{\theta}^{+}(r), & \psi_{-}=r^{j-} \phi_{\theta}^{-}(r),
\end{array}
$$

where $\phi_{\theta}^{ \pm}$are even entire functions with positive coefficients depending on $\theta$ :

$$
\phi_{\theta}^{ \pm}=\sum_{n=0}^{\infty} b_{n}^{ \pm}(\theta) r^{2 n}, \quad b_{0}^{ \pm}(\theta)=1 .
$$

Thus, the Fuchsian structure restricts the limiting behavior at the vacuum to one of two algebraic rates. The weak singularity $j_{+}$corresponds to waves that vanish identically at the vacuum, while the strong singularity $j_{-}$corresponds to waves with a finite limit at the vacuum that is not identically zero. Specifically, for weak waves the amplitude vanishes like the density:

$$
\begin{gathered}
\eta=a \psi_{+} e^{k u}, \\
a \psi_{+}=\text {const } \varrho^{(1-\theta) / 2} r^{(\theta+1) / 2 \theta} \phi_{+}=\text {const } \varrho \phi_{+},
\end{gathered}
$$

where the constants depend upon $k$. For strong waves the amplitude approaches a constant depending on $k$ :

$$
\begin{gathered}
\eta=a \psi_{-} e^{k u}, \\
a \psi_{-}=\operatorname{const} \varrho^{(1-\theta) / 2} r^{(1-\theta) / 2 \theta} \phi_{-}=\text {const } \phi_{-} .
\end{gathered}
$$

We shall see below that the space of entropy pairs $(\eta, q)$ is partitioned into two subspaces according to the structure of the Cauchy data at the vacuum state $V=\{(\varrho, u): \varrho=0\}$.

For now we simply mention that familiar weak pairs are provided by the mass, momentum and mechanical energy,

$$
(\varrho, \varrho u),\left(\varrho u, \varrho u^{2}+p\right),\left(\frac{1}{2} \varrho u^{2}+\varrho \varepsilon, \frac{1}{2} \varrho u^{3}+\varrho \varepsilon u+p u\right) .
$$

Familiar strong pairs are provided by the constant state, the velocity and pseudomechanical energy,

$$
(1,1),\left(u, u^{2} / 2+\tau\right),\left(\frac{1}{2} u^{2}+\sigma, u^{3} / 3+u(\sigma+\tau)\right) .
$$


Here the enthalpy $\tau$ and its transform $\sigma$ are defined by $\tau^{\prime}=p^{\prime} / \varrho, \sigma-\varrho \sigma^{\prime}=\tau$. The classical derivation of these pairs proceeds by rewriting the basic equations in the form of mass and velocity conservation $\varrho_{t}+(\varrho u)_{x}=0, u_{t}+\left(u^{2} / 2+\tau\right)_{x}=0$, which is legitimate for smooth flows, and then using multipliers $\sigma^{\prime}$ and $u$ to derive the law

$$
\left(\frac{1}{2} u^{2}+\sigma\right)_{t}+\left(u^{3} / 3+u(\sigma+\tau)\right)_{x}=0 .
$$

A systematic procedure for generating complete sequences of weak and strong waves will be provided below. We note here that the first three members of each sequence occur classically.

Next we shall construct the four families of Lax progressing waves [14]. We recall that for strictly hyperbolic systems of two equations (2.1) one may construct on each compact subset of the state space $R^{2}$ four families of entropy pairs with the following asymptotic expansion

$$
\begin{array}{ll}
\eta=e^{ \pm k w} \sum_{n=0}^{\infty} V_{n}^{ \pm} / k^{n}, & q=e^{ \pm k w} \sum_{n=0}^{\infty} H_{n}^{ \pm} / k^{n}, \\
\eta=e^{ \pm k z} \sum_{n=0}^{\infty} \tilde{V}_{n}^{ \pm} / k^{n}, & q=e^{ \pm k z} \sum_{n=0}^{\infty} \tilde{H}_{n}^{ \pm} / k^{n},
\end{array}
$$

where the variable coefficients $V, H$ are determined by the choice of the Riemann invariant phase. For convenience we shall regard $k$ as a positive parameter. The ratio of entropy flux to entropy is asymptotically equal to the corresponding eigenvalue: with the choice (2.15) we have $q / \eta=\lambda_{2}+\mathcal{O}(1 / k)$ since $H_{0}^{ \pm}=\lambda_{2} V_{0}^{ \pm}$; with the choice (2.16) we have $q / \eta=\lambda_{1}+\mathcal{O}(1 / k)$ since $\tilde{H}_{0}^{ \pm}=\lambda_{1} \tilde{V}_{0}^{ \pm}$. Unfortunately, the coefficients of the formal expansion diverge as the eigenvalues $\lambda_{1}$ and $\lambda_{2}$ coalesce. For gas dynamics the eigenvalues coalesce at the vacuum state $V$ (which represents a line of parabolic degeneracy since the eigenvectors also coalesce). We shall show below that although the formal series degenerates at the vacuum, the separable progressing waves introduced above remain bounded up to the vacuum and maintain an eigenspeed ratio

$$
q / \eta=\lambda+\mathcal{O}(1 / k)
$$

uniformly on $\varrho \geqq 0$.

To this end we recall that the Fuchsian equation (2.11) admits two independent solutions $\psi_{1}$ and $\psi_{2}$ which behave exponentially at infinity like the solutions to the equation with $\mu=0$ :

$$
\begin{aligned}
\psi_{1} e^{-r}=1+\mathcal{O}(1 / r), & \psi_{1}^{\prime} / \psi_{1}=1+\mathcal{O}(1 / r), \\
\psi_{2} e^{r}=1+\mathcal{O}(1 / r), & \psi_{2}^{\prime} / \psi_{2}=-1+\mathcal{O}(1 / r),
\end{aligned}
$$

as $r$ approaches infinity. By appropriately scaling solutions we may write $\psi_{1}=\psi_{+}$, $\psi_{2}=\psi_{-}+\psi_{+}$, since $\psi_{ \pm}$are independent. With this notation we introduce the entropy

$$
\eta=a \psi_{+} e^{k u} \equiv a \psi_{+} e^{-r} e^{k w},
$$

and regard it as a product of a density dependent amplitude with an exponential phase, $\eta=\sigma_{k}(\varrho) e^{k w}, \sigma_{k}=a \psi_{+} e^{-r}$. Thus the leading coefficient $V_{0}=a$, and we have 
$\eta=e^{k w}(a+\mathcal{O}(1 / k))$, uniformly on compact subsets of $\varrho>0$. In order to establish (2.17) we recast the expression (2.14) for the corresponding flux in the form

$$
q=\eta\left(\lambda_{2}+c\left(\psi_{+}^{\prime} / \psi_{+}-1\right)-(1+\theta) / 2 k\right),
$$

and observe that the second term may be written

$$
c\left(\psi_{+}^{\prime} / \psi_{+}-1\right)=\text { const } r\left(\psi_{+}^{\prime} / \psi_{+}-1\right) k \text { since } c=\text { const } r / k .
$$

The factor $r\left(\psi_{+}^{\prime} / \psi_{+}-1\right)$ is uniformly bounded as a consequence of (2.17) and the fact that differentiation reduces the order of an algebraic function by one. We conclude that entropy (2.19) satisfies $q / \eta=\lambda_{2}+\mathcal{O}(1 / k)$ on $\varrho \geqq 0$.

In a similar way one shows that the entropy

$$
\eta=a \psi_{+} e^{-k u}=a \psi_{+} e^{-r} e^{-k z}
$$

satisfies $\eta=e^{-k z}(a+\mathcal{O}(1 / k))$ on compact subsets of $\varrho>0$ and establishes a uniform ratio $q / \eta=\lambda_{1}+\mathcal{O}(1 / k)$ by rewriting the corresponding flux

in the form

$$
q=\eta\left(u-c \psi_{+}^{\prime} / \psi_{+}-(1+\theta) / 2 k\right)
$$

$$
q=\eta\left(\lambda_{1}+c\left(1-\psi_{+}^{\prime} / \psi_{+}\right)-(1+\theta) / 2 k\right) .
$$

In order to adopt convenient terminology we shall refer to a progressing wave as outgoing (incoming) if it is an increasing (decreasing) function of the phase. Since we have normalized $k$ to be positive, the incoming wave of the first field and the outgoing wave of the second field are weak. The companion waves which are outgoing in the first and incoming in the second field may be introduced as follows. The entropy

$$
\eta=a\left(\psi_{-}-\psi_{+}\right) e^{k u}=a\left(\psi_{-}-\psi_{+}\right) e^{r} e^{k z}
$$

satisfies $\eta=e^{k z}(a+\mathcal{O}(1 / k))$, on compact subsets of $\varrho>0$ and represents a strong (outgoing) wave of the first field; the coefficient $a \psi_{-}$does not vanish on $V$ and the eigenspeed ratio $q / \eta=\lambda_{1}+\mathcal{O}(1 / k)$ is uniform on $\varrho \geqq 0$. The entropy

$$
\eta=a\left(\psi_{-}-\psi_{+}\right) e^{-k u}=a\left(\psi_{-}-\psi_{+}\right) e^{r} e^{-k w}
$$

satisfies $\eta=e^{-k w}(a+\mathcal{O}(1 / k))$, on a compact subset of $\varrho>0$ and represents a strong incoming wave of the second field, i.e. $q / \eta=\lambda_{2}+\mathcal{O}(1 / k)$, uniformly in $\varrho \geqq 0$.

In order to apply compensated compactness it is necessary to analyze the limiting behavior of the second derivative of entropy at $V$ in order to determine whether or not the divergence of the corresponding entropy field remains in a compact subset of $H^{-1}$ as $\varepsilon$ vanishes. We shall briefly review the relevant calculations of the entropy production. Consider a diffusion process $u_{t}+f(u)_{x}=\varepsilon u_{x x}$ with $u \in R^{2}$. Premultiplication by the gradient of $\eta$ yields

$$
\nabla \eta\left(u_{t}+\nabla f u_{x}\right)=\varepsilon \nabla \eta u_{x x}, \quad \eta_{t}+q_{x}=\varepsilon \eta_{x x}-\nabla^{2} \eta u_{x}^{2} .
$$

Integration over the strip $[0, T]$ yields an expression for the change in total entropy:

$$
\varepsilon \int_{0}^{T} \int \nabla^{2} \eta u_{x}^{2} d x d t=\int \eta(x, 0) d x-\int \eta(x, T) d x
$$


If $\eta$ is non-negative and convex one obtains, in the standard fashion, a coercive bound $\varepsilon \iint \nabla^{2} \eta u_{x}^{2} d x d t \leqq c$, which implies that $\eta_{t}+q_{x}$ lies in a compact subset of $H^{-1}$ if $u_{\varepsilon}$ is uniformly bounded. In order to entertain solutions which approach a constant state $\bar{u}$ at infinity, one need only introduce a normalized entropy pair

$$
\begin{gathered}
\tilde{\eta}=\eta(u)-\eta(\bar{u})-\nabla \eta(\bar{u})(u-\bar{u}), \\
\tilde{q}=q(u)-q(\bar{u})-\nabla \eta \nabla f(\bar{u})(u-\bar{u}),
\end{gathered}
$$

which is obtained from a given pair $(\eta, q)$ by subtracting the linear part of $\eta$ at $\bar{u}$, and then repeating the argument above.

In the setting of gas dynamics we recall that the mechanical energy $\eta_{m}=\frac{1}{2} \varrho u^{2}$ $+\varrho \varepsilon(\varrho)$ is a strictly convex function of the densities of mass and momentum, $\varrho$ and $m=\varrho u$, provided that $p^{\prime}>0$. The corresponding flux $q$ is determined by the rate at which the pressure supplies work modulo mechanical transport, $q_{m}=u \eta_{m}+u p$. We shall see that the Hessian of the mechanical energy dominates the Hessian of weak waves in the sense that for each weak wave $\eta,\left|\nabla^{2} \eta\right| \leqq$ const $\nabla^{2} \eta_{m}$ for an appropriate constant. Here symmetric matrices are ordered in the standard fashion. It follows that the divergence of all the weak pairs lies in a compact subset of $H^{-1}$ and consequently that

$$
\left\langle v, \eta_{1} q_{2}-\eta_{2} q_{1}\right\rangle=\left\langle v, \eta_{1}\right\rangle\left\langle v, q_{2}\right\rangle-\left\langle v, \eta_{2}\right\rangle\left\langle v, q_{2}\right\rangle
$$

for all weak pairs $\left(\eta_{j}, q_{j}\right)$, where $v$ is a representing measure. Unfortunately, the Hessian of mechanical energy does not dominate the Hessian of strong waves and it remains to determine whether or not the divergence of a strong entropy field remains in a compact subset of $H^{-1}$ as $\varepsilon$ vanishes. If one could prove this for both weak and strong pairs then the argument in [10] could easily be adapted to show that $v$ reduces to a point mass.

Next we shall provide the relevant estimates on the Hessian of entropy. In considering the second derivative of $\eta_{m}$ we recall that the specific internal energy $\varepsilon$ is determined from the first law

$$
T d S=d \varepsilon+p d v,
$$

which yields, in the isentropic case the formula

$$
\varepsilon=\int^{\varrho} p / \varrho^{2} d \varrho .
$$

Since we shall work with artificial viscosity in the primitive variables $\varrho$ and $m$, all functions will be viewed in $(\varrho, m)$ coordinates:

$$
\begin{aligned}
r_{1} & =\left(1, \lambda_{1}\right)=(1, m / \varrho-c), \\
r_{2} & =\left(1, \lambda_{2}\right)=(1, m / \varrho+c), \\
\eta_{m}=\frac{1}{2} m^{2} / \varrho & +\tau(\varrho), \text { where } \tau=\varrho \int p / \varrho^{2} d \varrho .
\end{aligned}
$$

Since the eigenvectors $r_{j}$ are always biorthogonal in the inner product induced by $\nabla^{2} \eta$, i.e. $\nabla^{2} \eta(u)\left(r_{j}(u), r_{k}(u)\right) \equiv 0$, only two quantities need to be computed, namely $\nabla^{2} \eta\left(r_{j}, r_{j}\right)$ for $j=1,2$. A brief computation yields

$$
\nabla^{2} \eta_{m}\left(r_{j}, r_{j}\right)=\tau^{\prime \prime}+p^{\prime} / \varrho=2 p^{\prime} / \varrho
$$


for $j=1,2$. We note in passing that (2.20) demonstrates the known fact that hyperbolicity is equivalent to the convexity of mechanical energy, i.e. $\eta_{m}$ is convex if and only if $p$ is increasing. The lack of velocity dependence is a reflection of Galilean invariance. For a polytropic gas one obtains the formula

$$
\nabla^{2} \eta_{m}\left(r_{j}, r_{j}\right)=\varrho^{2 \theta-1}=\varrho^{\nu-2},
$$

which displays the behavior explicitly in terms of the adiabatic exponent $\gamma$.

The analysis of the Hessian of a progressing wave is facilitated by using the factored form. For concreteness, consider a progressing wave of the second field $\eta=\sigma(\varrho) e^{k w}$. The product formula

$$
\nabla^{2}(f g)=f \nabla^{2} g+2 \nabla f \vee \nabla g+g \nabla^{2} f
$$

leads easily to the decomposition

$$
e^{-k w} \nabla^{2} \eta=k^{2} \sigma \nabla w \otimes \nabla w+k \sigma \nabla^{2} w+2 k \nabla w \vee \nabla \sigma+\nabla^{2} \sigma,
$$

where the wedge symbol denotes the symmetric part of the tensor product: $a \vee b$ $=\frac{1}{2} a \otimes b+\frac{1}{2} b \otimes a$. The decomposition (2.21) is motivated by Lax [14] and indicates the relative contributions of amplitude and phase. The technical advantage of (2.21) stems from the ease with which the eigenderivatives of a Riemann invariant can be computed. We have

$$
\begin{gathered}
r_{1} \cdot \nabla w=0, \\
\nabla w \otimes \nabla w\left(r_{1}, r_{1}\right)=0, \quad \nabla w \otimes \nabla w\left(r_{2}, r_{2}\right)=4 \varrho^{2(\theta-1)}, \\
\nabla^{2} w\left(r_{1}, r_{1}\right)=(\theta+1) \varrho^{\theta-2}, \quad \nabla^{2} w\left(r_{2}, r_{2}\right)=(\theta-3) \varrho^{\theta-2} .
\end{gathered}
$$

We remark in passing that the invariants $w$ and $-z$ are not convex but quasiconvex functions of $\varrho$ and $m$. This point is discussed further below in connection with the construction of invariant quadrants for the Cauchy problem.

We shall begin with weak progressing waves and consider for concreteness the entropy $\eta=a \psi_{+} e^{-r} e^{k w}=\sigma_{k}(\varrho) e^{k w}$. The evenness of the analytic part $\phi_{+}$of $\psi_{+}$leads to the estimate

$$
\begin{aligned}
a \psi_{+} e^{-r} & =a r^{(\theta+1) / 2 \theta} \phi_{+} e^{-r}=\operatorname{const} \varrho\left(1-r+\mathcal{O}\left(r^{2}\right)\right) \\
& =\operatorname{const}\left(\varrho-k \varrho^{\theta} / \theta+\mathcal{O}\left(\varrho^{2 \theta+1}\right)\right),
\end{aligned}
$$

where the constants depend upon $k$. Substituting $\sigma=\varrho-k \varrho^{\theta} / \theta+\mathcal{O}\left(\varrho^{2 \theta+1}\right)$ into (2.21) reveals the dominant term near the vacuum $e^{-k w} \nabla^{2} \eta\left(r_{j}, r_{j}\right)=\mathcal{O}\left(\varrho^{2 \theta-1}\right)$. We remark that it is necessary to use the exact formula for the second term in $\sigma$; as in the case of the mechanical energy, the term $\varrho^{\theta-1}$ does not appear because of a cancellation. A similar analysis for weak waves of the first fields show that $\eta=a \psi_{+} e^{-r} e^{-k z}$ satisfies $e^{-k z} \nabla^{2} \eta\left(r_{j}, r_{j}\right)=\mathcal{O}\left(\varrho^{2 \theta-1}\right)$. In contrast, the Hessian of strong wave behaves like $\varrho^{\theta-2}$. The strong incoming wave of the second field $\eta=a\left(\psi^{-}-\psi^{+}\right) e^{-r} e^{-k 2}$ has a dominant coefficient

$$
a \psi^{-} e^{-r}=\text { const } \phi_{-} e^{-r}=\text { const }\left(1+r+\mathcal{O}\left(r^{2}\right)\right) .
$$


Substituting $\sigma=1+k \varrho^{\theta} / \theta+\mathcal{O}\left(\varrho^{2 \theta}\right)$ into (2.21) yields

$$
\begin{aligned}
& e^{k w} \nabla^{2} \eta\left(r_{1}, r_{1}\right)=-2 k \varrho^{\theta-2}+\mathcal{O}\left(\varrho^{2 \theta-2}\right), \\
& e^{k w} \nabla^{2} \eta\left(r_{2}, r_{2}\right)=2 k \varrho^{\theta-2}+\mathcal{O}\left(\varrho^{2 \theta-2}\right) .
\end{aligned}
$$

In a similar fashion it can be shown that the strong outgoing wave of the first field $\eta=a\left(\psi_{-}-\psi_{+}\right) e^{r} e^{k z}$ satisfies

$$
\begin{aligned}
& e^{-k z} \nabla^{2} \eta\left(r_{1}, r_{1}\right)=-2 k \varrho^{\theta-2}+\mathcal{O}\left(\varrho^{2 \theta-2}\right), \\
& e^{-k z} \nabla^{2} \eta\left(r_{2}, r_{2}\right)=2 k \varrho^{\theta-2}+\mathcal{O}\left(\varrho^{2 \theta-2}\right) .
\end{aligned}
$$

We remark that the saddle structure of the strong waves at the vacuum is anticipated by the knowledge that the only invariant regions for gas dynamics are given by quadrants of the form $\{(w, z): w \leqq$ const, $z \geqq$ const $\}$. If the signs of the leading terms in (2.22) and (2.23) were equal, there would exist additional local invariant regions with opposite orientation near the vacuum.

Progressing entropy waves may also be introduced with the aid of the Darboux integral representation of the solution to the Cauchy problem for (2.8) with data on the singular line $V$. In the case of a polytropic gas, the wave equation (2.9) has a classical power dependence

$$
\eta_{\varrho \varrho}=\varrho^{\gamma-3} \eta_{u u} .
$$

In the case $\gamma \geqq 3$, the Cauchy problem on $V$,

$$
\lim _{\varrho \rightarrow 0} \eta(\varrho, u)=\alpha(\varrho), \quad \lim _{\varrho \rightarrow 0} \eta_{\varrho}(\varrho, u)=\beta(\varrho),
$$

has an explicit integral representation [27, Chap. 2]. We remark in passing that the well-known uncoupling of the modes which occurs for $\gamma=3$ is reflected in the fact that (2.24) reduces to the constant coefficient wave equation. In characteristic coordinates the Cauchy data for the EPD equation takes the form

$$
\begin{gathered}
\lim _{w \rightarrow z \rightarrow 0} \eta(w, z)=\alpha(w), \\
\lim _{w \rightarrow z \rightarrow 0} a(w-z)^{b}\left(\eta_{w}-\eta_{z}\right)=\beta(w), \quad a=\left(\frac{\gamma+1}{4}\right)^{b}, \quad b=\frac{\gamma-3}{\gamma+1} .
\end{gathered}
$$

Weak waves correspond to the choice $\alpha=0$ while strong waves correspond to the choice $\beta=0$. The integral representation for weak waves is given by

$$
\eta=\mathrm{const} \int_{z}^{w}\{(w-z)(s-z)\}^{-\lambda} \beta(s) d s, \quad-\lambda=\frac{1}{2} \frac{(3-\gamma)}{(\gamma-1)},
$$

where the constant depends only on $\gamma$, [27]. In the case $1<\gamma \leqq 3$, the Cauchy problem for (2.24) is not well-posed on $V$. Nevertheless, the formula (2.25) provides a valid representation for solutions $\eta$ which vanish on $V$ and have a finite normal derivative $\beta$ :

$$
\lim _{\varrho \rightarrow 0} \eta(\varrho, u)=0, \quad \lim _{\varrho \rightarrow 0} \eta_{\varrho}(\varrho, u)=\beta(\varrho) .
$$

On the other hand, in the range $1<\gamma<3$, strong waves have a finite limit on $V$ but an infinite normal derivative. 
For notational convenience we shall write weak waves in the form $\eta=\int K^{-\lambda} g(s) d s$, where the kernel $K(w, z, s)$ is defined as follows,

$$
K=\{(w-s)(s-z)\}^{-\lambda} \quad \text { if } \quad z<s<w, \quad K=0 \quad \text { otherwise. }
$$

For computational convenience we shall confine attention to members of the physical sequence of adiabatic exponents corresponding to an odd number of degrees of freedom:

$$
\gamma=1+2 / n, \quad n=2 m+1, \quad m \geqq 1 .
$$

In this case the index $-\lambda$ of the EPD equation reduces to the positive integer $m$ and the kernel $K$ reduces to a polynomial. Correspondingly, for the special sequence (2.26) the hypergeometric Riemann function for EPD reduces to a Legendre polynomial. We shall not make use of integral representations with the Riemann function in this paper.

We observe that the change of scale $s=\tau w+(1-\tau) z$, recasts $(2.25)$ in a form which is convenient for introducing progressing waves, namely

$$
\eta=(w-z)^{2 m+1} \int_{0}^{1}\{\tau(1-\tau)\}^{m} g(\tau) d \tau
$$

The choice $g=D^{2 m} \psi$ leads to simple formulas for finitely generated progressing waves in the following manner. Using the chain rule we have

$$
\eta=(w-z) \int_{0}^{1} K_{m}(\tau)(w-z)^{2 m} D^{2 m} \psi(\tau w+(1-\tau) z) d \tau,
$$

where $K_{m}=\{\tau(1-\tau)\}^{m}$. An $m$-fold integration by parts may be carried out without producing boundary terms,

$$
\eta=(w-z) \int_{0}^{1} D^{m} K_{m}(\tau) D^{m} \psi d \tau .
$$

An additional $m$-fold integration by parts yields a progressing wave of order $m-1$ based on $\psi$ :

$$
\eta=P_{m} \psi=\sum_{j=0}^{m} a_{j}(w-z)^{m-j} C_{m-j-1} \psi,
$$

where the modes are coupled through a linear differential operator $C_{k}$ of order $k$,

$$
\begin{aligned}
C_{k} \psi & =D^{k} \psi(w)+(-1)^{k} D^{k} \psi(z), \quad k \geqq 1, \\
C_{-1} \psi & =\int_{z}^{w} \psi(x) d x .
\end{aligned}
$$

The fact that weak waves contain fronts involving both phases $w$ and $z$ is a central difficulty in trying to prove that the representing measure reduces to a point mass. On a fixed compact set in $\varrho>0$, one of the two modes may dominate, but in a neighborhood of $V$ both play a role. In Sect. 3 we shall employ waves of the form (2.27) with special choices of the base function $\psi$.

We emphasize that there exist a variety of solution representations each with distinct technical advantages. One could for example use the Darboux repre- 
sentation to prove that the Hessian of mechanical energy $\eta_{m}$ dominates the Hessian of all weak waves $\eta$ in the sense that

$$
\left|\nabla^{2} \eta\left(r_{j}, r_{j}\right)\right| \leqq \text { const } \nabla^{2} \eta_{m}\left(r_{j}, r_{j}\right),
$$

where the constant depends on the $C^{2}$ norm of $g$. The essential features of the proof that $v$ reduces to a point mass involves just a few estimates on the behaviour of solutions to the compatibility condition (2.2) near $V$. For a polytropic gas they can be readily verified using separation of variables or an integral representation. Presumably corresponding estimates will hold for equations of state which behave near $V$ like a polytropic gas.

We conclude this section by presenting a generalization of a classical result concerning entropy sequences for the equations of shallow water waves. We recall that by interpreting the variable $\varrho$ as height, the equations of isentropic gas dynamics with $\gamma=2$ assume the form of the equations of shallow water waves and admit a sequence of polynomial entropies in $(\varrho, u)[28, \mathrm{p} .460]$. This generalizes to the statement that for any $\gamma$-law gas the progressing entropy waves serve as a generating function for complete sequences of entropies which are polynomials in the sum and difference of the Riemann invariants modulo a factor of the density $\varrho$. These sequences may be introduced as follows. By scaling the wave

$$
\eta=a \psi_{+} e^{k u}=(k / \theta)^{(\theta+1) / 2 \theta} \varrho \phi_{+}(r) e^{k u},
$$

we find an entropy, $\eta=\varrho \phi_{+}(r) e^{k u}, r=k \varrho^{\theta} / \theta$ which depends analytically on the parameter $k$. Similarly by scaling $\eta=a \psi_{-} e^{k u}$, we find an entropy $\eta=\phi_{-}(r) e^{k u}$, which depends analytically on $k$. The power series expansions

$$
\begin{gathered}
\eta=\varrho \phi_{+} e^{k u}=\sum a_{n}^{+}(\varrho, u) k^{n}, \\
\eta=\phi_{-} e^{k u}=\sum a_{n}^{-}(\varrho, u) k^{n},
\end{gathered}
$$

generate homogeneous polynomial entropies with positive coefficients, namely

$$
a_{n}^{+}=\varrho \sum \alpha_{s r}^{+} \tau^{s} u^{r}, \quad a_{n}^{-}=\sum \alpha_{s r}^{-} \tau^{s} u^{r},
$$

where the summation is taken over indices $s$ and $r$ such that $s+r=n$ and $s$ is even, while $r=(w+z) / 2$ and $\tau=(w-z) / 2=\varrho^{\theta} / \theta$. The flux $q_{n}^{+}$corresponding to the weak entropy $a_{n}^{+}$, and the flux $q_{n}^{-}$corresponding to the strong entropy $a_{n}^{-}$have a similar structure:

$$
\begin{gathered}
q_{n}^{+}=\varrho \sum \beta_{s r}^{+} \tau^{s} u^{r}, \\
q_{n}^{-}=\sum \beta_{s r}^{-} \tau^{s} u^{r},
\end{gathered}
$$

which can be derived using (2.14) and the formula for the corresponding flux.

\section{The Representing Measure}

We shall consider a $\gamma$-law gas and prove that if $v$ is a compactly supported probability measure satisfying

$$
\left\langle v, \eta_{1} q_{2}-\eta_{2} q_{1}\right\rangle=\left\langle v, \eta_{1}\right\rangle\left\langle v, q_{2}\right\rangle-\left\langle v, \eta_{2}\right\rangle\left\langle v, q_{1}\right\rangle,
$$


for all smooth entropy pairs $\left(\eta_{j}, q_{j}\right)$ vanishing on the vacuum state $V=\{(w, z): w=z\}$, then either $v$ is a point mass or $v$ is concentrated on $V$. Following the terminology of Sect. 2 we shall refer to a pair $(\eta, q)$ which vanishes on $V$ as a weak pair. We shall restrict attention to adiabatic exponents with an odd number of degrees of freedom $\gamma=1+2 / n, n=2 m+1$ for technical convenience. We shall show that $v=\delta$ if $m \geqq 2$. The special case $m=1$ corresponds to a monotonic gas $\gamma=5 / 3$ and represents a transition index for the regularity of solutions to EPD. In the propositions below we shall assume $m \geqq 1$, with the exception of the last two, where we assume $m \geqq 2$ in order to appeal to enhanced regularity of progressing waves.

For notational convenience we shall abbreviate (3.1) in the form

$$
v \circ B=B \circ v,
$$

where the basic antisymmetric form $B$ is regarded as acting in entropy pairs or expected values of entropy pairs. We include the obvious remark that if (3.1) holds for all smooth weak pairs then it folds for all pointwise limits of smooth weak pairs.

The basic task in proving existence for conservation laws,

using the viscosity method,

$$
g(u)_{t}+f(u)_{x}=0,
$$

$$
g(u)_{t}+f(u)_{x}=\varepsilon D(u)_{x x}
$$

and compensated compactness is to prove that $g$ and $f$ commute with the operation of taking expected values with the representing measures $v_{(x, t)}$ corresponding to the sequence $u^{\varepsilon}$; as a consequence of a hypothesized or established uniform $L^{\infty}$ estimate for the solutions $u^{\varepsilon}$ of (3.4), one obtains that the divergence of the expected value of the field $(g, f)$ vanishes, i.e. $\bar{g}_{t}+\bar{f}_{x}=0$, where

$$
\bar{g}(x, t)=\int g(\lambda) d v_{(x, t)}, \quad \bar{f}(x, t)=\int f(\lambda) d v_{(x, t)},
$$

and $\lambda$ denotes the generic variable of the state space. If

$$
\bar{g}=g(\bar{u}) \quad \text { and } \quad \bar{f}=f(\bar{u}),
$$

then the center of mass $\bar{u}=\int \lambda d v_{(x, t)}(\lambda)$, provides a bounded distributional solution of (3.3). Since the equations of gas dynamics

$$
\begin{gathered}
\varrho_{t}+(\varrho u)_{x}=0, \\
(\varrho u)_{t}+\left(\varrho u^{2}+p(\varrho)\right)_{x}=0,
\end{gathered}
$$

are formally satisfied at the vacuum, it is sufficient to show that either $v$ is a point mass or $v$ is concentrated on $V$ in order to verify (3.5). Our strategy will be to prove that supp $v$ is contained in a set of the form $V \cup P$, where $P$ is an arbitrary point in $\varrho>0$. If $v$ admits the decomposition $v=v \mid V+\alpha \delta_{p}$, then the commutativity relation (3.1) applied to weak pairs yields

$$
\alpha\left(\eta_{1} q_{2}-\eta_{2} q_{1}\right)(P)=\alpha^{2}\left(\eta_{1} q_{2}-\eta_{2} q_{1}\right)(P),
$$

from which we deduce that either $\alpha=1$ and $v$ is a point mass or $\alpha=0$ and $v$ is concentrated on $V$. 
To this end we shall study regularity properties of the projections of $v$ onto the $w-z$ axes. Consider the smallest triangle

$$
\Omega=\left\{(w, z): w \leqq w_{0}, z \geqq z_{0}, w \geqq z\right\},
$$

which contains the support of a fixed $v$ satisfying (3.1). Let the three vertices be denoted by $P=\left(w_{0}, z_{0}\right), Q=\left(w_{0}, w_{0}\right), R=\left(z_{0}, z_{0}\right)$. By Galilean invariance one may assume that $\Omega$ is centered at the origin, i.e. that $w_{0}+z_{0}=0$. Let $P_{w} v$ and $P_{z} v$ denote the orthogonal projections of $v$ onto the horizontal segment $R P$ parallel to the $w$-axis and the vertical segment $P Q$ parallel to the $z$-axis, respectively:

$$
\begin{gathered}
P_{w} v(a, b)=v\{(w, z): a<w<b\}, \\
P_{z} v(a, b)=v\{(w, z): a<z<b\} .
\end{gathered}
$$

For notational convenience we shall regard $P_{w} v$ and $P_{z} v$ as acting on functions of $w$ and $z$ respectively. We shall show that the Lebesgue derivative of the projections of the weighted measure $\tilde{v}=(w-z)^{2 m} v$ vanish except possibly at the vertex $P$, i.e. $D P_{w} \tilde{v}=0$ for $z_{0}<w<w_{0} ; D P_{z} \tilde{v}=0$ for $z_{0}<z<w_{0}$. It follows that $P_{w} \tilde{v}$ and $P_{z} \tilde{v}$ vanish on the open segments $Q P$ and $P R$ respectively and that the support of $v$ is contained in $V \cup P$. We remark in passing that if one could also prove that either $D P_{w} \tilde{v}\left(w_{0}\right)=0$ or $D P_{z} \tilde{v}\left(z_{0}\right)=0$ then $\tilde{v}$ would vanish, i.e. $v$ would be concentrated on $V$.

We recall that the Lebesgue derivative

$$
D \mu\left(x_{0}\right)=\lim _{n \rightarrow \infty} 2 n \mu\left(x_{0}-1 / n, x_{0}+1 / n\right),
$$

of a non-negative measure $\mu$ exists at almost all points $x_{0}$ with respect to Lebesgue measure $d x$. If $D \mu$ vanishes a.e. with respect to $d x$ then $\mu$ is singular with respect to $d x$. If $D \mu$ vanishes a.e. with respect to $\mu$ then $\mu$ itself vanishes [29, Chap. 8]. Here it is convenient to regard the derivative as the limit of expected values of step waves:

$$
\begin{gathered}
D \mu\left(x_{0}\right)=\lim _{n \rightarrow \infty} \int s_{n}(x) d \mu, \\
s_{n}(x)=2 n \quad \text { if } \quad\left|x-x_{0}\right|<1 / n ; \quad s_{n}(x)=0 \quad \text { otherwise. }
\end{gathered}
$$

We shall differentiate the projections of $\tilde{v}$ using a sequence of weak EPD waves which approximate a step wave in each mode. In contrast to the constant coefficient wave equation, the modes of the EPD equation have varying amplitude. This difficulty is overcome by appealing to the coercive structure of special EPD waves in the nullspace of the Goursat operator.

To this end we shall first discuss the singular Goursat problem for (2.8) which poses data on characteristic rays issuing from $V$. Without loss of generality we shall work with the rays $w=0$ and $z=0$ issuing from the origin and prove that $D P_{w} \tilde{v}(0)=D P_{z} \tilde{v}(0)=0$. For this purpose we shall introduce special Goursat waves corresponding to a point source at the origin. Consider a consequence of smooth functions $g_{n}$ which approach the $\delta$-function at the origin, and which have, for simplicity, compact support. The Darboux formula provides a sequence of smooth weak entropies

$$
\eta_{n}=\int_{z}^{w}\{(w-s)(s-z)\}^{m} g_{n}(s) d s
$$


that converge pointwise to $\eta_{0}=(-z w)^{m} X$, where $X$ denotes the characteristic function of the fourth quadrant $w>0, z<0$. The associated flux is given by

$$
q_{0}=\frac{(n-1)}{2 n}\left\{(-z)^{m} w^{m+1}+w^{m} z(-z)^{m}\right\} X .
$$

We remark that in general the flux $q$ of a weak pair is easily computed using either of the formulas

$$
\begin{aligned}
& q=\lambda_{2} \eta-\frac{(\gamma+1)}{4} \int_{z}^{w} \eta(x, z) d x, \\
& q=\lambda_{1} \eta-\frac{(\gamma+1)}{4} \int_{z}^{w} \eta(w, y) d y,
\end{aligned}
$$

which may be derived by integrating the EPD equation,

$$
\begin{aligned}
& \left(q-\lambda_{2} \eta\right)_{w}=\lambda_{2 w} \eta=\frac{(\gamma+1)}{4} \eta, \\
& \left(q-\lambda_{1} \eta\right)_{z}=\lambda_{1 z} \eta=\frac{(\gamma+1)}{4} \eta,
\end{aligned}
$$

along horizontal segments joining $(z, z)$ to $(w, z)$ and vertical segments joining $(w, z)$ to $(w, w)$ respectively. One could, of course, begin with a weak entropy $\eta$ satisfying (2.8) and show by direct computation that the function $q$ supplied by (3.6a) or (3.6b) provides the corresponding flux.

In a similar fashion, a sequence of test functions $g_{n}$ approaching the derivative of the $\delta$-function at the origin generates the pair

$$
\begin{gathered}
\eta_{1}=(-z w)^{m-1}(w+z) X, \\
q_{1}=\frac{(n-1)}{n}(-z w)^{m-1}\left\{\frac{w^{2}}{2}+\frac{\left(n^{2}-2 n-1\right)}{(n-1)^{2}} w z+\frac{n^{2}}{2}\right\} X,
\end{gathered}
$$

as the pointwise limit of smooth weak pairs. We remark that both $G_{0}=\left(\eta_{0}, q_{0}\right)$ and $G_{1}=\left(\eta_{1}, q_{1}\right)$ lie in the nullspace of the Goursat operator, i.e. vanish on the characteristic rays $w=0$ and $z=0$. A complete set of waves in the nullspace of the Goursat operator may be formally generated by using Galilean invariance: if $\eta$ solves EPD so does $\eta_{w}+\eta_{z}$. For the purposes of this paper we shall only need the two independent pairs $G_{0}$ and $G_{1}$.

A basic coercive property of the Goursat waves $G_{0}$ and $G_{1}$ is expressed by a sign condition on the form $B$, namely

$$
B\left(G_{0}, G_{1}\right)=\eta_{0} q_{1}-\eta_{1} q_{0}=\frac{2}{n(n-1)}(-z w)^{2 m} X \geqq 0,
$$

which is obtained by direct substitution. Additional coercive properties will be discussed below; for the moment we shall use (3.7) to derive a regularity property of $v$. Our analysis is motivated in part by the following lemma.

Lemma 3.1. If $v$ is not concentrated on $V$ then the vertex $P$ of the minimal triangle $\Omega$ lies in the support of $v$. 
Proof. We shall argue by contradiction using the outgoing 2-waves and the incoming 1-waves which were introduced in Sect. 2 and satisfy

$$
\begin{array}{ll}
\eta_{1}=e^{-k z}(a+\mathcal{O}(1 / k)), & \eta_{2}=e^{k w}(a+\mathcal{O}(1 / k)), \\
q_{1}=\eta_{1}\left(\lambda_{1}+\mathcal{O}(1 / k)\right), & q_{2}=\eta_{2}\left(\lambda_{2}+\mathcal{O}(1 / k)\right) .
\end{array}
$$

Consider the normalized form of (3.1),

$$
\frac{\left\langle v, \eta_{1} q_{2}-\eta_{2} q_{1}\right\rangle}{\left\langle v, \eta_{1}\right\rangle\left\langle v, \eta_{2}\right\rangle}=\frac{\left\langle v, q_{2}\right\rangle}{\left\langle v, \eta_{2}\right\rangle}-\frac{\left\langle v, q_{1}\right\rangle}{\left\langle v, \eta_{1}\right\rangle} .
$$

The integrand in the numerator of the left satisfies

$$
\eta_{1} q_{2}-\eta_{2} q_{1}=e^{k(w-z)}\left\{\left(\lambda_{1}-\lambda_{2}\right) a+\mathcal{O}(1 / k)\right\} .
$$

If the vertex $\left(w_{0}, z_{0}\right)$ does not lie in $\operatorname{supp} v$ then

$$
\left\langle v, \eta_{1} q_{2}-\eta_{2} q_{1}\right\rangle \mid \leqq \text { const } e^{k\left(w_{0}-z_{0}-\delta\right)}
$$

for an appropriate $\delta$. On the other hand, for every $\varepsilon>0$, the expected values in the denominator satisfy

$$
\left\langle v, \eta_{1}\right\rangle \geqq \text { const }_{\varepsilon} e^{-k\left(z_{0}+\varepsilon\right)} ; \quad\left\langle v, \eta_{2}\right\rangle \geqq \text { const } e^{k\left(w_{0}-\varepsilon\right)},
$$

since $\Omega$ is minimal. Thus, as $k$ approaches infinity through an appropriate subsequence, the left side of (3.8) vanishes while the right side converges to the difference of averaged values of the characteristic speeds,

$$
\begin{gathered}
0=\bar{\lambda}_{2}-\bar{\lambda}_{1}, \\
\bar{\lambda}_{2}=\lim _{k \rightarrow \infty} \frac{\left\langle v, q_{2}\right\rangle}{\left\langle v, \eta_{2}\right\rangle}, \quad \bar{\lambda}_{1}=\lim _{k \rightarrow \infty} \frac{\left\langle v, q_{1}\right\rangle}{\left\langle v, \eta_{1}\right\rangle} .
\end{gathered}
$$

Since the characteristic speeds are nondecreasing functions of the characteristic speeds,

$$
\lambda_{1_{w}}=\lambda_{2_{z}}=(3-\gamma) / 4 \geqq 0,
$$

$\gamma \leqq 3$, the extreme values satisfy

$$
\max _{R P} \lambda_{1}=\lambda_{1}(P) \geqq \bar{\lambda}_{1} ; \quad \bar{\lambda}_{2} \geqq \lambda_{2}(P)=\min _{P Q} \lambda_{2},
$$

and strict hyperbolic produces the contradiction $\bar{\lambda}_{2} \geqq \lambda_{2}(P)>\lambda_{1}(P) \geqq \bar{\lambda}_{1}$.

Lemma 3.1 is the first step in proving that $v$ is concentrated at $P$ if it is not concentrated on $V$. The second step is to establish regularity in the form of uniform boundedness of expected values of singular waves. Suppose that $\psi$ is an arbitrary smooth compactly support function on the line. Fix $m$ and consider the progressing pair of order $m-1$ based on $\psi$ :

$$
\left(\eta_{n}, q_{n}\right), \quad n_{n}=P_{m}\left(\psi_{n}\right), \quad \psi_{n}=n \psi(n x) .
$$

Lemma 3.2. For each $\psi$ the expected values $\left\langle v, \eta_{n}\right\rangle$ and $\left\langle v, q_{n}\right\rangle$ are bounded in $n$.

This lemma is motivated by the technical observation that the difference between entropy flux and characteristic speed times entropy is a smoothing operator on weak pairs: 


$$
\begin{gathered}
q-\lambda_{j} \eta=\tau_{j}(\eta) \\
\tau_{1}=-\frac{(\gamma+1)}{4} \int_{z}^{w} \eta(x, z) d x, \quad \tau_{2}=\frac{(\gamma+1)}{4} \int_{z}^{w} \eta(w, y) d y .
\end{gathered}
$$

The regularization afforded by the form $B(\eta, q ; \hat{\eta}, \hat{q})=\eta \hat{q}-\eta \hat{q}$, is put into evidence by the identity $B=\tau_{j} \hat{\eta}-\hat{\tau}_{j} \eta$, which involves one order of integration on the right side. The basic observation is that the left side of (3.1) is generally more regular than the right side, an imbalance which can only be resolved if $v$ is a point mass. We shall begin to quantify these remarks with a proposition that is needed in the proof of Lemma 3.2.

Proposition 3.3. Suppose $(\eta, q)$ is a pair which vanishes to order $m-1$ on the characteristic rays through the origin in the sense that $|\eta|+|q| \leqq$ const $|z w|^{m-1}$. Then for all smooth compactly supported functions $\psi$, the form $B_{n}=\eta q_{n}-\eta_{n} q$ is bounded uniformly in $n$.

Proof. Assume for concreteness that $\psi$ is supported on $(-1,1)$ and consequently that $\psi_{n}$ is supported on $(-1 / n, 1 / n)$. The waves $\left(\eta_{n}, q_{n}\right)$ vanish on

$$
F=\{(w, z): w<-1 / n \text { or } z>1 / n\}
$$

by finite propagation speed and the main action is confined to the characteristic strips

$$
S_{w}=\{(w, z):|w|<1 / n\}, \quad S_{z}=\{(w, z):|z|<1 / n\}
$$

We do not repeat the standing condition $w \geqq z$. We shall partition $F^{c}$ in four regions involving an interaction zone $I$ and a wake quadrant $Q$ :

$$
\begin{gathered}
F^{c}=\tilde{S}_{w} \cup \tilde{S}_{z} \cup Q \cup I, \\
Q=\{(w, z): w \geqq 1 / n, z \leqq-1 / n\}, \quad I=\{(w, z):|w| \leqq 1 / n,|z| \leqq 1 / n\}, \\
\tilde{S}_{w}=S_{w} \cap I^{c}, \quad \tilde{S}_{z}=S_{z} \cap I^{c} .
\end{gathered}
$$

On the interaction zone $I$ the pair $(\eta, q)$ is small, $|\eta|+|q| \leqq$ const $/ n^{2 m-2}$, and one sees easily that $B_{n}$ is uniformly bounded on $I$. On the wake quadrant, $\eta_{n}$ reduces to constant function $\eta_{n}=a_{m} \int \psi_{n}(x) d x$, which is clearly bounded uniformly in $n$, while $q_{n}$ reduces to a constant function which can be shown to be uniformly bounded by integrating (3.6) by parts. Thus, we need only consider the truncated strips $\tilde{S}_{w}$ and $\tilde{S}_{z}$ on which $\eta_{n}$ consists of just a single mode:

$$
\begin{gathered}
\eta_{n}=\sum_{j=0}^{m} a_{j}(w-z)^{m-j} D_{m-j-1} \psi_{n}(w) \text { on } \tilde{S}_{w}, \\
\eta_{n}=\sum_{j=0}^{m} a_{j}(w-z)^{m-j}(-1)^{m-j-1} D_{m-j-1} \psi_{n}(z) \text { on } \tilde{S}_{z} .
\end{gathered}
$$

By substituting (3.9a) into (3.6a) we find that

$$
\begin{gathered}
q_{n}=\lambda_{2} \eta+\mathcal{O}\left(n^{m-1}\right), \\
B_{n}=\eta\left(\lambda_{2} n_{n}+\mathcal{O}\left(n^{m-1}\right)\right)-\eta_{n}\left(\lambda_{2} \eta+\mathcal{O}\left(n^{-m}\right)\right)=\mathcal{O}\left(n^{1-m} n^{m-1}+n^{m} n^{-m}\right) .
\end{gathered}
$$

on the set $\tilde{S}_{w_{\tilde{s}}}$ In a similar fashion, by substituting $(3.9 \mathrm{~b})$ into (3.6b) we find that $B_{n}=\mathcal{O}(1)$ on $\tilde{S}_{z}$. 
Proof of Lemma 3.2. Fix $(\eta, q)$ and consider the commutativity relation

$$
\left\langle v, \eta q_{n}-\eta_{n} q\right\rangle=\langle v, \eta\rangle\left\langle v, q_{n}\right\rangle-\left\langle v, \eta_{n}\right\rangle\langle v, q\rangle \text {. }
$$

Suppose, on the contrary that

$$
\overline{\lim }\left|\left\langle v, \eta_{n}\right\rangle\right|=\infty \quad \text { and/or } \quad \overline{\lim }\left|\left\langle v, q_{n}\right\rangle\right|=\infty \text {. }
$$

By dividing (3.10) by the appropriate factor $\left\langle v, \eta_{n}\right\rangle$ or $\left\langle v, q_{n}\right\rangle$ it follows that there exists constants $\alpha$ and $\beta$ not both zero such that $0=\alpha\langle v, \eta\rangle-\beta\langle v, q\rangle$, since the left side is bounded uniformly in $n$. We conclude that the expected value of $B$ acting on weak pairs $(\eta, q),(\hat{\eta}, \hat{q})$ of order $(z w)^{m-1}$ vanishes:

$$
\langle v, \eta \hat{q}-\hat{\eta} q\rangle=\langle v, \eta\rangle\langle v, \hat{q}\rangle-\langle v, \hat{\eta}\rangle\langle v, q\rangle=0 .
$$

Substituting $G_{0}$ and $G_{1}$ into (3.11) leads to the contradiction that the vertex $P=\left(w_{0}, z_{0}\right)$ does not lie in supp $v$. We have

$$
\begin{gathered}
0=\left\langle v, \eta_{0} q_{1}-\eta_{1} q_{0}\right\rangle=\left\langle v \mid Q, \eta_{0} q_{1}-\eta_{1} q_{0}\right\rangle, \\
\left\langle\eta_{0} q_{1}-\eta_{1} q_{0}\right\rangle>0 \text { on } Q=\{(w, z): w>0, z<0\} .
\end{gathered}
$$

Next we shall prove that the derivatives of the projections of the weighted measure $\tilde{v}$ exist and vanish escept possibly at the vertex $P$ of $\Omega$. As we remarked above, Galilean invariance allows one to label the point in question as the origin. We shall show that $D P_{w} \tilde{v}(0)=D P_{z} \tilde{v}(0)=0$, using the coercive character of $B$ on the subspace of progressing waves $P_{m}(\psi)$. To this end we shall show that the sum of appropriate bilinar forms of the type $B_{n}=\eta_{n} \hat{q}_{n}-n_{n} q_{n}$, can be constructed to approximate a step wave in each of the characteristic strips $S_{w}$ and $S_{z}$ and deduce, using the uniform boundedness of $B_{n}$, that the operation

$$
\lim _{n \rightarrow \infty}\left\langle v, \sum_{j} B_{n}^{j}\right\rangle
$$

produces the derivative of $\tilde{v}$ modulo a factor.

We shall begin by estimating the leading term of $B_{n}$. Suppose that $\psi$ and $\hat{\psi}$ are supported on $(-1,1)$.

Lemma 3.4. The form $B_{n}=B_{n}(\psi, \hat{\psi})$ admits the power decompositions

$$
\begin{aligned}
& B_{n}=\sum_{j=0}^{2 m} n^{2 m-j-1}(w-z)^{2 m-j} A_{m-j}(n w) \text { on } \tilde{S}_{w}, \\
& B_{n}=\sum_{j=0}^{2 m} n^{2 m-j-1}(w-z)^{2 m-j} A_{m-j}(n z) \text { on } \tilde{S}_{z},
\end{aligned}
$$

where the coefficient forms $A_{k}$ represent bilinear maps sending a pair of functions $(\psi, \hat{\psi})$ into a function $A_{k}(x)=A_{k}(\psi, \hat{\psi})(x)$, determined by the first $k-1$ derivatives of $\psi$ and $\hat{\psi}$. The leading coefficient is given by

$$
A_{m}=\operatorname{const}\left(D_{m-2} \psi D_{m-1} \tilde{\psi}-D_{m-2} \hat{\psi} D_{m-1} \psi\right) \text {. }
$$

Proof. For concreteness we shall consider the vertical strip $\tilde{S}_{w}$. We shall express $B_{n}$ in terms of the integral operator $\tau$ as follows,

$$
B_{n}=\tau_{n} \hat{\eta}_{n}-\hat{\tau}_{n} \eta_{n} ; \quad \tau=\tau(\eta)=\frac{(\gamma+1)}{4} \int_{z}^{w} \eta(x, z) d x
$$


On $\hat{S}_{w}$, the waves $\eta_{n}$ and $\hat{\eta}_{n}$ contain only one mode, e.g.

$$
\eta_{n}=\sum_{j=0}^{m} a_{j}(w-z)^{m-j} D_{m-j-1} \psi_{n}(w) .
$$

An explicit integration shows that the function

$$
\tau_{n} \equiv \tau\left(\eta_{n}\right)=\frac{(\gamma+1)}{4} \int_{-1 / n}^{w} \eta_{n}(x, z) d x
$$

satisfies

$$
\tau_{n}=\frac{(\gamma+1)}{4} a_{0}(w-z)^{m} D_{m-2} \psi_{n}(w)+\sum_{j=1}^{m} \mathcal{O}(w-z)^{m-j} n^{m-j-1} .
$$

Here lower order derivatives are crudely estimated by the formula,

$$
D_{k} \psi_{n}=n^{k+1} D_{k} \psi(n w)=\mathcal{O}\left(n^{k+1}\right) .
$$

Substituting (3.12) and (3.13) into $B_{n}$ together with the corresponding expressions for $\hat{\eta}_{n}=P_{m}\left(\hat{\psi}_{n}\right)$ and $\hat{\tau}_{n}=\tau\left(\hat{\eta}_{n}\right)$ yields the desired result once one observes that the homogeneous terms involving derivatives of order $m-2$ cancel due to symmetry. The product of $D_{m-2} \psi_{n}$ with $D_{m-2} \hat{\psi}_{n}$ does not appear.

The process of differentiating $\tilde{v}$ is based on the following uniform bound for the expected value of $B_{n}$ acting on progressing waves.

Lemma 3.5. For each smooth compactly supported pair $(\psi, \hat{\psi})$, the expected value

$$
\left\langle v, \sum_{j=0}^{2 m} n^{2 m-j-1}(w-z)^{2 m-j} A_{m-j}(\psi, \hat{\psi})\right\rangle
$$

is bounded uniformly in $n$. Here $A_{k}$ is evaluated at $n w$ if the variable $(w, z)$ lies in $S_{w}$ and at $n z$ if the variable $(w, z)$ lies in $S_{z}$.

Proof. Apply (3.1) to the waves $\eta_{n}=P_{m}\left(\psi_{n}\right), \hat{\eta}_{n}=P_{m}\left(\hat{\psi}_{n}\right)$. The right side is bounded by Lemma 3.2 while the integration on the left side takes place over the set $F^{c}$ introduced in Proposition 3.3:

$$
\begin{gathered}
\left\langle v, \eta_{n} \hat{q}_{n}-\hat{\eta}_{n} q_{n}\right\rangle=\left\langle v \mid F^{c}, \eta_{n} \hat{q}_{n}-\hat{\eta}_{n} q_{n}\right\rangle, \\
F^{c}=\tilde{S}_{w} \cup \tilde{S}_{z} \cup Q \cup I .
\end{gathered}
$$

Since the pairs $\left(\eta_{n}, q_{n}\right)$ and $\left(\hat{\eta}_{n}, \hat{q}_{n}\right)$ are uniformly bounded on the interaction zone $I$ and the wake quadrant $Q$, as was shown in the proof of Proposition 3.3, the quantity $\left\langle v \mid S, \eta_{n} \hat{q}_{n}-\hat{\eta}_{n} q_{n}\right\rangle$ is uniformly bounded. Here $S$ denotes the union of the characteristic strips $S_{w}$ and $S_{z}$. The desired bound follows from the power decomposition in Lemma 3.4.

The coercive character of $B$ acting on progressing waves can now be put into evidence by the statement that for each $(\psi, \hat{\psi})$ there exists a constant $c=c(\psi, \hat{\psi})$ so that

$$
\left\langle v \mid S_{n}, n^{2 m-1}(w-z)^{2 m} A_{2 m}(\psi, \hat{\psi})-c \sum_{j=1}^{2 m} n^{2 m-j-1}(w-z)^{2 m-j}\right\rangle \leqq c
$$


The form of the leading coefficient motivates the weight $(w-z)^{2 m}$. At this point we remark that a savings in technical detail can be obtained if one merely proves that the lower Lebesgue derivatives vanish, i.e. $\underline{D} P_{w} \tilde{v}=0$ for $w<w_{0}, \underline{D} P_{z} \tilde{v}=0$ for $z>z_{0}$, where $\underline{D} \mu(x)=\underline{\lim } 2 n \mu(x-1 / n, x+1 / n)$. Indeed, if the lower Lebesgue derivatives of a non-negative measure $\mu$ on the line vanish almost everywhere with respect to $\mu$ then $\mu$ vanishes [29, Chap. 8]. We shall show below that

$$
\underline{D} P_{w} \tilde{v}(0)=\underline{D}_{z} \tilde{v}(0)=0 \text {. }
$$

A similar analysis yields the vanishing of the upper Lebesgue derivative. However, that analysis is unnecessary for current purposes.

The basic strategy for estimating the lower derivative can be conveniently explained by considering a model situation where it is assumed that the restriction $v \mid S_{n}$ satisfies

$$
\left\langle v \mid S_{n}, n^{2 m-1}(w-z)^{2 m}-\sum_{j=1}^{2 m} c n^{2 m-j-1}(w-z)^{2 m-j}\right\rangle \leqq c,
$$

for some constant $c$. The model situation arises by replacing the leading coefficient $A_{2 m}(\psi, \hat{\psi})$ by the indicator function of $(-1,1)$. We shall show that one may choose a finite set of pairs $\left(\psi_{i}, \hat{\psi}_{i}\right)$ so that the sum of the leading coefficients,

$$
\sum_{i} A_{2 m}\left(\psi_{i}, \hat{\psi}_{i}\right)
$$

is positive on $(-1,1)$. Then the argument for the model situation can be applied with a simple modification to the bound obtained by considering (3.14) with the choice $\left(\psi_{i}, \hat{\psi}_{i}\right)$ and summing over $i$. In the following analysis we shall assume that the integer $m$ exceeds one.

Proposition 3.6. The uniform upper bound (3.16) implies that

$$
\lim \left\langle v \mid S_{n},(w-z)^{2 m} n\right\rangle=0,
$$

or equivalently that (3.15) holds.

Proof. We shall show that quantity $\left\langle v \mid S_{n},(w-z)^{2 m} n^{2 m-1}\right\rangle$ is uniformly bounded in $n$. We shall estimate the lower order terms of (3.16), namely,

$$
l_{n}=\sum_{j=1}^{2 m} c n^{2 m-j-1}(w-z)^{2 m-j}
$$

in two steps. If $(w-z) \leqq n^{-\alpha}$, then

$$
l_{n} \leqq 2 m c n^{2 m-2-(2 m-1) \alpha}
$$

and vanishes in the limit if the exponent is negative, i.e. if

$$
(2 m-2) /(2 m-1)<\alpha .
$$

If $(w-z) \geqq n^{-\beta}$ with $\beta<1$, then the leading $L_{n}=(w-z)^{2 m} n^{2 m-1}$ dominates the lower of terms in the sense that $L_{n} \geqq l_{n} / 2$ for large $n$. Therefore if we fix $\alpha$ so that $(2 m-2) /(2 m-1)<\alpha<1$, we find that an upper bound of the form 


$$
\begin{gathered}
\left\langle v \mid S_{n} \cap A_{n}, L_{n}-l_{n}\right\rangle+\left\langle v \mid S_{n} \cap A_{n}^{c}, L_{n}-l_{n}\right\rangle \leqq \text { const } \\
A_{n}=\left\{(w, z):(w-z) \leqq n^{-\alpha}\right\},
\end{gathered}
$$

yields the desired estimate, $\left\langle v \mid S_{n}, L_{n}\right\rangle \leqq$ const .

As a technical comment, we remark that in the case $m \geqq 2$ there is flexibility in the exponents. For example, if one assumes a weakened version of (3.16) in which the order of the leading coefficient is reduced by a small amount $\varepsilon$, i.e.,

$$
\left\langle v \mid S_{n}, n^{2 m-1}(w-z)^{2 m-\varepsilon}-\sum_{j=1}^{2 m} c n^{2 m-j-1}(w-z)^{2 m-j}\right\rangle \leqq c,
$$

then the statement (3.17) still holds. This fact makes the actual situation in which $A_{2 m}(\psi, \hat{\psi})$ has compact support easily accessible.

We conclude this section with a discussion of the structure of the leading coefficient

$$
\tilde{A}_{m} \equiv \text { const } A_{2 m}(\psi, \hat{\psi})=D_{m-2} \psi D_{m-1} \hat{\psi}-D_{m-2} \hat{\psi} D_{m-1} \psi \text {. }
$$

In the simplest case, $m=2$, the function $A_{2}$ reduces to the familiar form $\tilde{A}_{2}=\psi \hat{\psi}^{\prime}$ $-\hat{\psi} \psi^{\prime}=\psi^{2} D(\hat{\psi} / \psi)$. If $\psi$ has compact support and we define $\hat{\psi}$ in terms of $\psi$ by the rule $\hat{\psi}=x \psi$, then $\hat{\psi}$ has compact support and $A_{2}$ is coercive, $\tilde{A}_{2}(\psi, \hat{\psi})=\psi^{2} \geqq 0$. If $m=2$, it suffices to employ one pair $(\psi, x \psi)$, where $\psi$ is a rough approximation of the indicator function of $(-1,1)$ :

$$
\begin{array}{ll}
\psi(x) \geqq 0 & \text { for }-1 \leqq x \leqq 1, \\
\psi(x)>0 & \text { for }-1+\varepsilon \leqq x \leqq 1-\varepsilon, \\
\psi(x)=0 & \text { otherwise }
\end{array}
$$

with $\varepsilon$ small. If $m \geqq 2$, we introduce $\phi=D_{m-2} \psi$, and write

$$
\tilde{A}_{m}=\phi D \hat{\phi}=\hat{\phi} D \phi=\phi^{2} D(\hat{\phi} / \phi)=\left(D_{m-2} \psi\right)^{2} D\left(D_{m-2} \hat{\psi} / D_{m-2} \psi\right) \text {. }
$$

We observe that if $\psi$ is supported on $(-1,1)$ and satisfies

$$
\int_{-1}^{1} \psi(x) d x=0
$$

then the function $\hat{\psi}=x \psi-p \int_{-1}^{x} \psi(y) d y$ is supported on $(-1,1)$ and satisfies $D_{p} \hat{\psi}=x D_{p} \psi$. Hence $A_{m}$ is coercive on the pair $(\psi, \hat{\psi})$ :

$$
\tilde{A}_{m}(\psi, \hat{\psi})=\left(D_{m-2} \psi\right)^{2} \quad \text { if } \quad \hat{\psi}=x \psi-(m-2) \int_{-1}^{x} \psi d x
$$

By taking a finite collection of functions $\psi_{i}$ satisfying (3.18) and having distinct zeroes in $(-1,1)$ we may arrange that

$$
\begin{gathered}
\sum_{i} A_{2 m}\left(\psi_{i}, \hat{\psi}_{i}\right) \geqq 0 \text { on }-1 \leqq x \leqq 1, \\
\sum_{i} A_{2 m}\left(\psi_{i}, \hat{\psi}_{i}\right)>0 \text { on } \quad-1+\varepsilon \leqq x \leqq 1+\varepsilon .
\end{gathered}
$$

The remaining details are routine and are omitted. 


\section{The Viscosity Method}

We shall briefly discuss the existence problem for the parabolic system,

$$
\begin{gathered}
\varrho_{t}+m_{x}=\varepsilon \varrho_{x x}, \\
m_{t}+\left(m^{2} / \varrho+p\right)_{x}=\varepsilon m_{x x},
\end{gathered}
$$

and prove an $\varepsilon$-dependent lower bound on $\varrho$, cf. Theorem 4.1 . We shall begin with some elementary remarks concerning a priori estimates for systems of the form

$$
u_{t}+f(u)_{x}+g(u)=u_{x x},
$$

where $f$ and $g$ are arbitrary smooth maps on $R^{n}$. It is not difficult to show that a priori pointwise control on the amplitude leads to pointwise and integral control on the higher derivatives: if $|u(\cdot, t)|_{\infty} \leqq m\left(t,\left|u_{0}\right|_{\infty}\right)$, then

$$
\begin{gathered}
|u(\cdot, t)|_{c^{k}} \leqq p_{k}\left(t,\left|u_{0}\right|_{c^{k}}\right), \\
|u(\cdot, t)|_{H^{k}} \leqq q_{k}\left(t,\left|u_{0}\right|_{H^{k}}\right),
\end{gathered}
$$

where the bounds $p_{k}$ and $q_{k}$ denote finitely-valued functions depending on $f, g$, and $m$.

In the semilinear case, $f=0$, the estimate (4.3) can be derived by inverting the heat operator while the estimate (4.4) can be derived by differentiating (4.2) in space and multiplying by $D_{x}^{k} u$. In the quasilinear case the same process works after one derivative is put on the heat kernel. For example, if $g=0$, then

$$
u(x, t)=\int_{-\infty}^{\infty} Z(x-y, t) u_{0}(y) d y+\int_{0}^{t} \int_{-\infty}^{\infty} Z_{x}(x-y, t-\tau) f(u(y, \tau)) d y d \tau,
$$

where

$$
Z=\frac{1}{\{4 \pi(t-\tau))^{1 / 2}} \exp \left\{-(x-y)^{2} / 4(t-\tau)\right\} .
$$

A standard induction shows that the spatial $C^{k}$ norms satisfy a Gronwall inequality of the form

$$
\phi(t) \leqq \phi(0)+\int_{0}^{t} \frac{h(\tau)}{(t-\tau)^{1 / 2}} \phi(\tau) d \tau
$$

since the first spatial derivative of the kernel is integrable,

$$
\begin{gathered}
\left|Z_{x}\right| \leqq \frac{c}{(t-\tau)} \exp \left\{-(x-y)^{2} / 2(t-\tau)\right\}, \\
\int_{-\infty}^{\infty}\left|Z_{x}(x-y, t-\tau)\right| d y \leqq c(t-\tau)^{-1 / 2} .
\end{gathered}
$$

Given pointwise control of the form (4.3), one can derive integral control (4.4) with the standard multiplier $D_{x}^{k} u$. One may, of course, proceed in a variety of ways.

We recall that, in the setting of artificial viscosity applied to isentropic gas dynamics, the presence of invariant quadrants leads to a $L^{\infty}$ estimate which is independent of $\varepsilon$. The Riemann invariants $w=m / \varrho+\varrho^{\theta} / \theta$ and $-z=-m / \varrho+\varrho^{\theta} / \theta$ 
are quasi-convex functions of $(\varrho, m)$, i.e. convex in the directions tangential to their level sets:

$$
\begin{aligned}
& r_{1} \cdot \nabla w=0, \quad \nabla^{2} w\left(r_{1}, r_{1}\right) \geqq 0, \\
& r_{2} \cdot \nabla z=0, \quad-\nabla^{2} z\left(r_{2}, r_{2}\right) \geqq 0 .
\end{aligned}
$$

The general theory of invariant regions developed by Chueh, Conley and Smoller [30] yields upper bounds of the form

$$
w(x, t) \leqq \sup _{x} w_{0}(x), \quad-z(x, t) \leqq \sup _{x}\left\{-z_{0}(x)\right\} .
$$

The intersection of half-planes provides the classical invariant quadrant of the $w-z$ plane which translates in uniform control $|u| \leqq$ const, $0 \leqq \varrho \leqq$ const, where the constants depend only on the modulus of the data. Thus, to continue a local solution of (4.1) one needs only an a priori lower bound on the density $\varrho \geqq \delta(t, \varepsilon)>0$. We shall sketch a derivation of such a estimate using the $L^{\infty}$ bound and the dissipation of mechanical energy.

We recall from Sect. 2 that quasilinear systems of the form $u_{t}+f(u)_{x}=\varepsilon u_{x x}$ admit an estimate of the form

$$
\int_{-\infty}^{\infty} \tilde{\eta} d x+\varepsilon \int_{0}^{t} \int_{-\infty}^{\infty} \nabla^{2} \tilde{\eta} u_{x}^{2} d x d t \leqq \int_{-\infty}^{\infty} \eta_{0} d x
$$

for a normalized convex entropy $\tilde{\eta}=\eta(u)-\eta(\bar{u})-\nabla \eta(\bar{u})(u-\bar{u})$, provided that the solution $u$ approaches the constant state $\bar{u}$ at infinity. Applying (4.6) to the mechanical energy

$$
\frac{1}{2} m^{2} / \varrho+\sigma(\varrho) \equiv \frac{1}{2} m^{2} / \varrho+\varrho^{\gamma} / \gamma(\gamma-1)
$$

yields

$$
\int_{-\infty}^{\infty} \frac{1}{2} \varrho(u-\bar{u})^{2}+Q \sigma(\varrho, \bar{\varrho}) d x \leqq c,
$$

where $Q \sigma$ denotes the quadratic part of $\sigma$ at $\bar{\varrho}: Q \sigma=\sigma(\varrho)-\sigma(\bar{\varrho})-\sigma^{\prime}(\bar{\varrho})(\varrho-\bar{\varrho})$. In the following we shall assume without loss of generality that $\bar{u}=0$. With the aid of the integral estimate (4.7) we shall obtain a pointwise lower bound on $\varrho$ by deriving $H^{1}$ estimates on convex functions $h(\varrho)$ which blow up algebraically at the vacuum. At first we shall proceed formally and multiply the mass equation by $h^{\prime}$ and integrate over the strip $(0, t)$ :

$$
\begin{gathered}
h^{\prime} \varrho_{t}+h^{\prime}(\varrho u)_{x}=\varepsilon h^{\prime} \varrho_{x x} \\
h_{t}+(h \varrho u)_{x}-h^{\prime \prime} \varrho^{2} \varrho_{x} u=h_{x x}-\varepsilon h^{\prime \prime} \varrho_{x}^{2}, \\
\int_{-\infty}^{\infty} h(t)-h(0) d x+\varepsilon \int_{0}^{t} \int_{-\infty}^{\infty} h^{\prime \prime} \varrho_{x}^{2} d x d t=\int_{0}^{t} \int_{-\infty}^{\infty} h^{\prime \prime} \varrho^{2} \varrho_{x} u d x d t .
\end{gathered}
$$

If $M^{1 / 2}$ denotes an upper bound for $\varrho$, we may use Young's inequality to obtain

$$
\begin{gathered}
h^{\prime \prime} \varrho \varrho_{x} u \leqq \frac{\varepsilon}{2 M} h^{\prime \prime} \varrho^{2} \varrho_{x}^{2}+\frac{2 M}{2} h^{\prime \prime} \varrho^{2} u^{2}, \\
\int h(t)-h(0) d x+\frac{\varepsilon}{2} \iint h^{\prime \prime} \varrho_{x}^{2} \leqq \frac{2 M}{\varepsilon} \iint h^{\prime \prime} \varrho^{2} u^{2} .
\end{gathered}
$$


In order to estimate the right hand side of (4.9) in terms of the mechanical energy we shall restrict $h$ to the class $K$ of strictly convex $C^{2}$ functions with the following properties:

$$
\begin{gathered}
h(\bar{\varrho})=h^{\prime}(\bar{\varrho})=0 \\
h(\varrho)=\varrho^{-\alpha} \text { on }(0, \bar{\varrho} / 2) \quad \text { for some } \quad \alpha, 0<\alpha<1 .
\end{gathered}
$$

We note that the functions in $K$ have the following convenient properties

$$
\begin{gathered}
h \leqq c(\varrho-\bar{\varrho})^{2} \text { for } \varrho \text { near } \bar{\varrho} \\
\varrho^{2} h^{\prime \prime} \leqq c \varrho \text { for } \varrho / 2 \leqq \varrho \leqq M^{1 / 2}, \\
\varrho^{2} h^{\prime \prime} \leqq c h(\varrho) \text { for } 0<\varrho<\bar{\varrho} / 2 .
\end{gathered}
$$

Thus for a solution with velocity bounded by $M$ we have $h^{\prime \prime} \varrho^{2} u^{2} \leqq c\left(\varrho u^{2}+h\right)$, and a Gronwall inequality,

$$
\int h(t)-h(0) d x+\frac{\varepsilon}{2} \int_{0}^{t} \int h^{\prime \prime} \varrho_{x}^{2} d x d t \leqq \frac{c t}{\varepsilon}+\int_{0}^{t} \int h(\varrho) d x d t,
$$

since the contribution from the term $\varrho u^{2}$ is controlled by the mechanical energy. Therefore we obtain a growth estimate

$$
\int_{-\infty}^{\infty} h(t) d x+\int_{0}^{t} \int_{-\infty}^{\infty} h^{\prime \prime} \varrho_{x}^{2} d x d t \leqq c e^{c t / \varepsilon}
$$

where the constant $c$ depends upon $h$ and the amplitude and mechanical energy of the data. We shall convert the integral estimate into a pointwise lower bound using the following lemma (which has numerous obvious generalizations).

Lemma 4.1. If $\phi(t)$ is a non-negative function satisfying

$$
\begin{gathered}
\phi(t)-\phi(s) \geqq c_{1}(t-s)^{1 / 2} \quad \text { if } t>s, \\
\int_{0}^{T} \phi^{-\alpha} d t \leqq c_{2},
\end{gathered}
$$

then $\phi \geqq c_{3}$ on the interval $(0, T)$, where the constant $c_{3}$ depends on $c_{1}, c_{2}, T$, and $\alpha$.

First we shall verify (4.11) for the function

$$
\phi(t)=\min _{x} \varrho(x, t) .
$$

This can be done by inverting the heat operator in the mass equation to obtain

$$
\varrho=\int Z(x-y, t-s) \varrho(y, s) d y+\iint_{0}^{t} Z_{x}(x-y, t-\tau) u \varrho(y, \tau) d y d \tau=I_{1}+I_{2} .
$$

Since $Z$ is a non-negative fundamental solution we have

$$
I_{1} \geqq \min _{y} \varrho(y, s),
$$

while the bound (4.5) implies $\left|I_{2}\right| \leqq c(t-s)^{1 / 2}$. To prove (4.15) we shall show that

$$
\int_{0}^{t} \int_{-\infty}^{\infty} h^{2}+h_{x}^{2} d x d t \leqq c e^{c t / \varepsilon}
$$


from which the Sobolev inequality implies

$$
\int_{0}^{t} \sup _{x} h(\varrho(x, t)) d t \leqq c e^{c t / \varepsilon}
$$

or equivalently

$$
\int_{0}^{t} \sup _{x} \varrho^{-\alpha}(x, t) d t \leqq c e^{c t / \varepsilon}
$$

The first term in (4.13) can be handled by simply replacing $h$ by $h^{2}$ in (3.14). The second term can be handled by replacing $h$ with a function $g$ satisfying $h^{\prime 2} \leqq g^{\prime \prime}$, so that

$$
\iint h_{x}^{2} d x d t=\iint h^{\prime 2} \varrho_{x}^{2} d x d t \leqq \iint g^{\prime \prime} \varrho_{x}^{2} d x d t \leqq c e^{c t / \varepsilon} .
$$

We conclude that if $(\varrho, u)$ is a smooth solution with initial data $\left(\varrho_{0}, u_{0}\right)$ which approach a constant state $(\varrho, \bar{u})$ at infinity and satisfy

$$
\begin{gathered}
\varrho_{0} \geqq \delta>0, \\
\int_{-\infty}^{\infty}\left(\varrho_{0}-\bar{\varrho}\right)^{2}+(u-\bar{u})^{2} d x<\infty,
\end{gathered}
$$

where

$$
\bar{\varrho}=\lim _{x \rightarrow \pm \infty} \varrho_{0}(x), \quad \bar{u}=\lim _{x \rightarrow \pm \infty} u_{0}(x),
$$

then the density $\varrho$ is strictly positive, $\varrho(x, t) \geqq \delta(t)>0$. These a priori estimates may be used in a variety of ways to produce an existence theorem. One representative theorem is the following.

Theorem 4.1. Suppose that $\left(\varrho_{0}-\bar{\varrho}, u_{0}-\bar{u}\right) \in C^{2} \cap H^{2}$ and $\varrho_{0} \geqq \delta>0$. There exists $a$ global solution $(\varrho, u)$ to the Cauchy problem with data $\varrho_{0}, u_{0}$ such that

$$
\begin{gathered}
(\varrho(\cdot, t)-\bar{\varrho}, u(\cdot, t)-\bar{u}) \in C^{2} \cap H^{2}, \\
\varrho \geqq \delta(t)>0,
\end{gathered}
$$

for an appropriate function $\delta$.

One may, of course, consider regularity properties of the solution. It is easy to show that any locally bounded distributional solution to a system of the form (4.2) is $C^{\infty}$ if $f$ and $g$ are $C^{\infty}$.

\section{References}

1. Ball, J.M.: On the calculus of variations and sequentially weakly continuous maps. In: Proceedings Dundee Conference on Ordinary and Partial Differential Equations (1976). Lecture Notes in Mathematics, Vol. 564. Berlin, Heidelberg, New York: Springer 1976, pp. 13-25

2. Ball, J.M.: Convexity conditions and existence theorems in nonlinear elasticity. Arch. Rat. Mech. Anal. 63, 337-403 (1977)

3. Ball, J.M., Currie, J.C., Olver, P.J. : Null Lagrangians, weak continuity, and variational problems of arbitrary order. J. Functional Analysis 41, 135-175 (1981)

4. Bahkvarov, N.: On the existence of regular solutions in the large for quasilinear hyperbolic systems. Zh. Vychisl. Mat. Fiz. 10, 969-980 (1970) 
5. Dacorogna, B.: Weak continuity and weak lower semicontinuity of nonlinear functionals. Lefschetz Center for Dynamical Systems Lecture Notes, Vol. 81-77. Brown University 1981

6. Dacorogna, B.: A relaxation theorem and its applications to the equilibrium of gases. Arch. Rat. Mech. Anal. (to appear)

7. Dacorogna, B. : A generic result for nonconvex problems in the calculus of variations. J. Functional Analysis (to appear)

8. DiPerna, R.J.: Global solutions to a class of nonlinear hyperbolic systems of equations. Commun. Pure Appl. Math. 26, 1-28 (1973)

9. DiPerna, R.J.: Existence in the large for nonlinear hyperbolic conservation laws. Arch. Rat. Mech. Anal. 52, 244-257 (1973)

10. DiPerna, R.J.: Convergence of approximate solutions to conservation laws. Arch. Rat. Mech. Analysis (1983) (to appear)

11. Glimm, J.: Solutions in the large for nonlinear hyperbolic systems of equations. Commun. Pure Appl. Math. 18, 697-715 (1965)

12. Glimm, J., Lax, P.D.: Decay of solutions of systems of nonlinear hyperbolic conservation laws. Am.Math. Soc. $101(1970)$

13. Lax, P.D.: Hyperbolic systems of conservation laws. II. Commun. Pure Appl. Math. 10, 537-566 (1957)

14. Lax, P.D.: Shock waves and entropy. In: Contributions to nonlinear functional analysis, Zarantonello, E.A. (ed.). New York: Academic Press 1971, pp. 603-634

15. Lax, P.D., Wendroff, B.: Systems of conservation laws. Commun. Pure Appl. Math. 13, 217-237 (1960)

16. Murat, F.: Compacité par compensation. Ann. Scula Norm. Sup. Pisa Sci. Fis. Math. 5, 489-507 (1978)

17. Murat, F.: Compacité par compensation: Condition necessaire et suffisante de continuité faible sous une hypotheses de rang constant. Ann. Suola Norm. Sup. Pisa 8, 69-102 (1981)

18. Murat, F.: L'injection du cone positif de $H^{-1}$ dans $W^{-1, q}$ est compacte pour tout $q>2$ (preprint)

19. Nishida, T.: Global solutions for an initial boundary value problem of a quasilinear hyperbolic system. Proc. Jpn. Acad. 44, 642-646 (1968)

20. Nishida, T., Smoller, J.A.: Solutions in the large for some nonlinear hyperbolic conservation laws. Commun. Pure Appl. Math. 26, 183-200 (1973)

21. Tartar, L.: Une nouvelle méthode de résolution d'équations aux dérivées partielles nonlinéaires. In: Lecture Notes in Mathematics, Vol. 665. Berlin, Heidelberg, New York: Springer 1977, pp. $228-241$

22. Tartar, L.: Compensated compactness and applications to partial differential equations. In: Research notes in mathematics, nonlinear analysis, and mechanics: Heriot-Watt Symposium, Vol. 4, Knops, R.J. (ed.). New York: Pitman Press 1979

23. Tartar, L.: The compensated compactness method applied to systems of conservation laws. In: Systems of nonlinear partial differential equations, Ball, J.M. (ed.). NATO ASI Series, C. Reidel Publishing Co. (1983)

24. Isaacson, E.: Global solution to a Riemann Problem for a non-strictly hyperbolic system of conservation laws arising in enhanced oil recovery. J. Comp. Phys. (to appear)

25. Temple, B.: Global solution of the Cauchy problem for a class of $2 \times 2$ non-strictly hyperbolic conservation laws. Adv. Appl. Math. (to appear)

26. Keyfitz, B., Kranzer, H.: A system of non-strictly hyperbolic conservation laws arising in elasticity theory. Arch. Rat. Mech. Anal. 72 (1980)

27. Betsadze, A.V.: Equations of the mixed type. New York: Macmillan Company 1964

28. Whitham, G.B.: Linear and nonlinear waves. New York: Wiley 1974

29. Rudin, W.: Real and complex analysis. New York: McGraw-Hill 1966

30. Chueh, K.N., Conley, C.C., Smoller, J.A.: Positively invariant regions for systems of nonlinear diffusion equations. Ind. Univ. Math. J. 26, 372-411 (1977)

Communicated by A. Jaffe

Received April 4, 1983 